\title{
Large-eddy simulation of Rayleigh-Taylor turbulence with compressible miscible fluids
}

\author{
J. P. Mellado and S. Sarkar ${ }^{a)}$ \\ Department of Mechanical and Aerospace Engineering, University of California San Diego, \\ La Jolla, California 92093-0411 \\ Y. Zhou \\ Lawrence Livermore National Laboratory, University of California, Livermore, California 94551
}

(Received 25 March 2004; accepted 17 May 2005; published online 13 July 2005)

\begin{abstract}
Turbulence developed from Rayleigh-Taylor instability between two compressible miscible fluids in an unbounded domain is addressed in this paper. It is demonstrated that the turbulent Mach number in the turbulent core has an upper bound, independent of the density ratio under a broad range of initial mean configurations. The initial thermodynamic state of the system determines the amount of potential energy per unit mass involved in the turbulent mixing stage, and thus the characteristic level of turbulent fluctuations that is achievable is linked to the characteristic speed of sound such that the turbulent Mach number is limited. For the particular case of an ideal gas, this bound on the turbulent Mach number is found to be between 0.25 and 0.6, depending on the particular initial thermodynamic state. Hence, intrinsic compressibility effects (those owing to large Mach number) are likely to be limited in the turbulent stage of a pure Rayleigh-Taylor problem. This result is confirmed by large-eddy simulations (LES) of systems with density jumps at the interface of $3: 1$, a density ratio for which there is extensive data available in the literature. The LES predictions of the mixing depth growth and overall mixing agree with results previously obtained in incompressible configurations with a negligibly small Mach number, and the data fully describing the Reynolds stresses and the budget of the (resolved) turbulent kinetic energy equation are provided. (C) 2005 American Institute of Physics. [DOI: 10.1063/1.1965130]
\end{abstract}

\section{INTRODUCTION}

Rayleigh-Taylor instability occurs when the interface between two fluids with different densities is subjected to a pressure force $(-\nabla p)$ pointing toward the heavy fluid, and this instability can eventually lead to a turbulent flow. The standard configuration is the hydrostatic equilibrium under the presence of a volumetric force $\mathbf{g}$, directed toward the light fluid,

$$
\boldsymbol{\nabla} p=\rho \mathbf{g} .
$$

The problem was described by Rayleigh ${ }^{1}$ and the first linear stability analysis was carried out by Taylor, ${ }^{2}$ an analysis extended later to include diffusion effects ${ }^{3}$ and, more recently, compressibility effects. ${ }^{4}$ Interest in the topic reappeared strongly in 1980 s, due to the inertial confinement fusion (ICF) programs. $^{5-8}$ Literature covers theoretical, $^{3,4,7,9}$ experimental, $5,8,10,11$ and numerical ${ }^{6,8,12-15}$ approaches to the problem. A detailed comparison between experiments and simulations has also been documented. ${ }^{16,17}$

Qualitatively, the flow evolves as follows. ${ }^{6,7}$ If the perturbation of the interface is small enough, then the linear analysis is valid and describes the exponential growth in this linear stage. Then follows a nonlinear stage, where asymmetric structures in the form of rising bubbles and falling spikes start forming owing to the baroclinic production of

\footnotetext{
${ }^{a)}$ Author to whom correspondence should be addressed. Telephone: (858) 534-8243. Fax: (858) 534-7599. Electronic mail: ssarkar@mae.ucsd.edu
}

vorticity, organized in rings around the protruding fingers. This stage is shown in Fig. 1 (top row), where isosurfaces of the density field and the horizontal vorticity field are represented. The latter is defined by $\omega_{h}^{2}=\omega_{x}^{2}+\omega_{y}^{2}$ if the $O Z$ axis is chosen parallel to the volumetric force. The vortex rings are slightly distorted in the vertical direction due to the shear between adjacent fingers.

This organized distribution of coherent structures is eventually lost due to the nonlinear interaction among them, and more intertwined distributions of both fields are observed in the bottom row of Fig. 1. Larger structures appear, either by the amalgamation of smaller ones or by their presence in the initial condition. At the same time, KelvinHelmholtz instabilities appear at the sides of the fingers. By the end of this stage the memory of the initial conditions (at least "small scales") can be potentially lost, with the turbulent stage taking over. Larger scales continue to be formed, viscosity having little effect on them and nonlinearity driving their energy toward the smallest scales.

If the previous description holds and the statistics of the flow become truly independent of the initial conditions and viscosity, then the width of the mixing region between the two layers, denoted by $h(t)$, in the low Mach number case usually considered in the literature, depends only on $\rho_{L}, \rho_{H}$, $g$, and $t$. Thus, dimensional analysis yields $h / g t^{2}=f(A)$ which, for the case $A \ll 1$, can be approximated by the linear relationship 


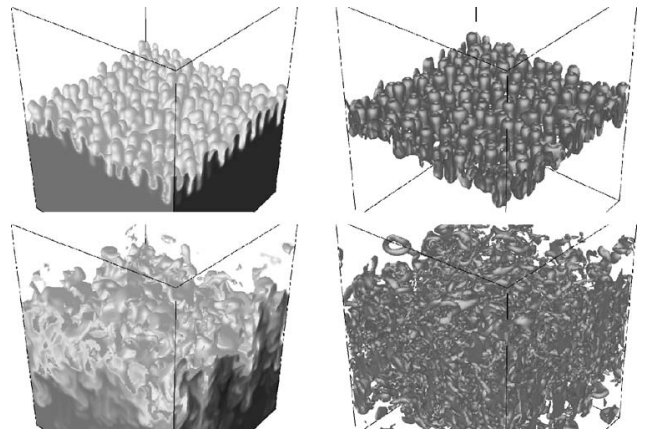

FIG. 1. Isosurfaces of density field (left), $\rho=2$, and of horizontal vorticity (right), $\omega_{h}=0.2$ of maximum. Top $-t=2.5 \tau$ (nonlinear stage); bottom- $t$ $=10 \tau$ (turbulent stage). The characteristic time $\tau=\sqrt{\delta_{\rho, 0} /(A g)}$ is defined using the initial thickness of the mixing layer. Gravity is acting downward. Only $1 / 4$ of the whole domain is shown for clarity.

$$
h=\alpha A g t^{2},
$$

the Atwood number being defined in terms of the ratio between the densities of the heavy fluid $\rho_{H}$ and the light fluid $\rho_{L}$ as

$$
A=\frac{\rho_{H} / \rho_{L}-1}{\rho_{H} / \rho_{L}+1} .
$$

This scaling leads to a temporal variation of the Reynolds number as $\operatorname{Re}_{h}=h \dot{h} / \nu \sim t^{3}, \nu$ being the kinematic viscosity, which implies that the Kolmogorov scale $\eta=\left(\nu^{3} / \varepsilon\right)^{1 / 4}$, where $\varepsilon$ is the mean dissipation rate of turbulent kinetic energy per unit mass, continually decreases with time ${ }^{13}$ as $\eta \sim t^{-1 / 4}$. Therefore, direct numerical simulation (DNS) is restricted in the temporal interval that can be used. As an alternative to DNS, the problem can be closed using numerics alone, for example, Youngs ${ }^{12}$ and Linden et al. ${ }^{16}$ solved the Euler equations with a monotone scheme. Cook et al. ${ }^{15}$ performed large-eddy simulation (LES) employing a subgrid eddy diffusivity that is strongly nonlinear, being dependent on the eighth-order derivative of the velocity. Here we adopt the LES strategy as well, but using a dynamic mixed model, already demonstrated to provide the temporal evolution of the large-scale three-dimensional fields with reasonable accuracy in different flows. ${ }^{18}$ The reader is referred to any of the several review articles available in the literature ${ }^{18-21}$ for a general description of the LES approach.

A large extent of the research on the Rayleigh-Taylor problem has focused on the growth of the mixing zone. The total thickness $h$ is usually split into the bubble penetration distance $h_{b}$ and the spike penetration height $h_{s}$. According to the literature, $\alpha_{b}$ seems to be independent of the Atwood number, whereas $\alpha_{s}$ exhibits a slight increase with it, causing asymmetry in the flow for sufficiently large density ratios. A recent thorough review of the available data ${ }^{22}$ shows $\alpha_{b}$ varying between 0.03 and 0.08 , the numerical simulations generally indicating smaller growth rates than the experimental results. In this regard, the strong influence that largescale initial conditions (large meaning comparable to the final mixing thickness) may have in the subsequent flow development has been reported by Linden et al. ${ }^{16}$ and Cook et al., ${ }^{13}$ among others.
However, an analysis of the role of compressibility in the turbulent stage of the Rayleigh-Taylor problem has not been reported. This paper considers this turbulent stage with miscible fluids in an unbounded domain. As mentioned before, work has been done on the effect of compressibility on the initial linear stage, an example for immiscible fluids being provided by Livescu, ${ }^{4}$ but our paper is concerned with the stage of fully developed turbulence. A priori, these compressibility effects could appear if the speed of sound of the fluid is sufficiently reduced (e.g., reducing the reference pressure of the system) so that a characteristic Mach number is sufficiently large, and this is the motivation for the present study. It should be noted that the Richtmyer-Meshkov problem is fundamentally different in this respect, since the velocity scale, imposed there by the impulsive deposition of vorticity at the front, can be set independently of the thermodynamic state.

A general overview containing the latest results on compressible turbulence can be found in the work by Chassaing et $a .^{23}$ In the case of free shear flows, there is a strong intrinsic compressibility effect: the growth rate of the shear layer thickness and the turbulence intensity are significantly reduced as the Mach number increases. The cause has been the subject of a considerable amount of study during the past years, ${ }^{24-29}$ leading to the following picture: the production term in the turbulent kinetic energy equation reduces as a consequence of the decrease in the pressure-strain correlation, which diminishes the transfer of energy from the streamwise fluctuations to the cross-stream fluctuations. It is reasonable, then, to formulate the same questions for the Rayleigh-Taylor problem, where the input of energy is essentially different. Is the growth rate $\alpha$ of the mixing depth $h$ a function of the Mach number? And if so, is the physical mechanism the same as in the case of a shear flow?

The focus of this paper is on intrinsic compressibility, density variations due to pressure variations, that is measured by the flow Mach number. There is no mean flow in the problem, and the compressibility associated with the turbulent fluctuations is measured by the turbulent Mach number,

$$
M_{t}=\frac{q}{\langle c\rangle},
$$

where $q=\sqrt{2 K}, K$ being the turbulent kinetic energy, and $\langle c\rangle$ the average speed of sound. The average of any variable $\phi$ is written as $\langle\phi\rangle$ and it is computed as a plane average at a fixed inhomogeneous location $z$. They denote Reynolds averages for quantities $\phi$ per unit volume and Favre averages for quantities $\phi$ per unit mass, unless otherwise stated. The quantity $M_{t}(z)$ varies across the mixing zone. The value of $M_{t}$ in the core is of interest where the speed of sound is that of the mixed interfacial fluid, $c_{0}$.

The compressibility of the fluid also modifies the stability inside of the fluid layers with respect to the incompressible situation. This stability is measured by the buoyancy-frequency ${ }^{30,31}$ 


$$
N^{2}=\frac{g}{\theta} \frac{d \theta}{d z}
$$

with $\theta$ being the potential temperature. For an ideal gas, it follows that

$$
N^{2}=g \frac{\rho}{p^{1 / \gamma}} \frac{d}{d z}\left(\frac{p^{1 / \gamma}}{\rho}\right),
$$

where $p$ is the pressure, $\rho$ is the density, and $\gamma$ is the ratio of specific heats. The coordinate $z$ increases in the opposite direction of the volumetric force $\mathbf{g}$. The buoyancy-frequency has been written in terms of the pressure and the density because it is more suitable for the analysis to be presented below. The sign of $N^{2}$ determines the static stability of the configuration. Therefore, in addition to the Rayleigh-Taylor instability at the interface between the two layers originating from the density jump, each layer can individually be buoyancy-stable if $N^{2}>0$ or buoyancy-unstable if $N^{2}<0$. Although the topic of the investigation reported here is intrinsic compressibility in a turbulent flow, the buoyancycompressibility coupling may affect the evolution of Rayleigh-Taylor turbulence and is included in the analysis. Note that one could also study the evolution of small (nonturbulent) fluctuations in cases with positive, negative, and zero values of $N^{2}$, but this is not what is being done here.

The paper is organized as follows. In Sec. II, the problem is defined and a theoretical analysis of the role of compressibility, giving definite bounds on the turbulent Mach number, is presented. The dynamic mixed model used to close the subgrid terms in the filtered equations is presented in Sec. III along with the general formulation of the problem. Sections IV and V contain the results from the LES, confirming the theoretical predictions obtained in Sec. II. Several statistics, such as mean profiles, Reynolds stresses, and the budget of the resolved turbulent kinetic energy, are also discussed.

\section{COMPRESSIBILITY OF THE TURBULENCE}

We consider the hydrostatic equilibrium of a layer of heavy fluid on top of a layer of lighter fluid. Choosing the $O Z$ axis parallel to and in the opposite direction of the constant volumetric force $\mathbf{g}$, the equilibrium is determined by

$$
\frac{d p}{d z}=-\rho g .
$$

The function $\rho=\rho(p)$ has to be provided in order to solve the problem, and different configurations will be explored below.

The equation of state for a mixture of two ideal gases reads

$$
\frac{p}{\rho}=\frac{R^{0} T}{W}=R^{0} T\left(\frac{Y_{L}}{W_{L}}+\frac{Y_{H}}{W_{H}}\right),
$$

where $Y_{i}$ denotes the species mass fraction of the fluid $i$ and $R^{0}$ is the universal gas constant. The molecular weight of the mixture, $W$, has been written in terms of the molecular weight of the heavy fluid and the light fluid, $W_{H}$ and $W_{L}$, respectively.
The intrinsic compressibility is determined by the turbulent Mach number, defined by Eq. (4). The local speed of sound of an ideal gas is given by

$$
c=\sqrt{\gamma \frac{R^{0} T}{W}}=\sqrt{\gamma \frac{p}{\rho}},
$$

where $\gamma$, the ratio of specific heats, lies in the range $1<\gamma$ $<5 / 3$.

With respect to the turbulent kinetic energy, turbulent theory predicts and experiments confirm that, in the incompressible limit, a self-similar state is achieved after a sufficiently long time. A thorough dimensional analysis is presented in the work by Cook et al. ${ }^{13}$ [see Eq. (6) therein], which yields

$$
q_{0}=\beta \sqrt{A g h}
$$

as a characteristic scale of the turbulent velocity fluctuations at each time. In this expression, $A g$ represents the constant external force per unit mass, with $A$ being the Atwood number and $h(t)$ the thickness of the increasing mixing depth. With miscible fluids, an (smaller) effective Atwood number instead of the nominal Atwood number $A$ would be more appropriate, as discussed by Cook et al. ${ }^{15}$ The coefficient $\beta$, of order 1 , has to be provided by the experimental data.

A priori, it is not known how Eq. (10) is modified by compressibility. Hence an analysis based on the available potential energy is preferred to study the problem and Eq. (10) is not used. However, the fact that the fluid velocity is determined by the potential energy per unit mass (set by the initial thermodynamic variables) links the maximum of $q_{0}$ over the time to $c_{0}$, the characteristic speed of sound, such that $M_{t}$ is bounded from above. The consequence is that $M_{t}$, small at early times because of an initial state that is steady, might not become large enough for intrinsic compressibility effects to be strong, so that Eq. (10) might be reasonably well satisfied even in the compressible case of RayleighTaylor turbulence.

This section is devoted to obtain analytically an upper bound on $M_{t}$ for three different initial configurations $\rho(p)$ leading to the three different types of static stability: (1) constant $\rho / p$ in each layer, in Sec. II A, which is buoyancystable $\left(N^{2}>0\right)$; (2) constant $\rho / p^{1 / \gamma}$ in each layer, in Sec. II B, which is buoyancy-neutral $\left(N^{2}=0\right)$, a pure Rayleigh-Taylor problem; (3) constant $\rho$ in each layer, in Sec. II C, which is buoyancy-unstable $\left(N^{2}<0\right)$.

\section{A. Two buoyancy-stable layers}

This section considers two layers which are buoyancystable, i.e., a relation $\rho(p)$ in each of them such that the buoyancy-frequency is positive. The particular configuration, shown in Fig. 2, is formed by two layers, each composed of a pure homogeneous fluid. The ratio between the molecular weight and the temperature of the mixture varies between two well-defined levels, $W_{L} / T_{L}$ at the bottom and $W_{H} / T_{H}$ (larger) at the top. This setup implies a relation $\rho \propto p$, and Eq. (6) provides $N^{2}>0$. This case can be easily set up, for instance, by depositing a layer of heavy fluid on top of a second lighter fluid at the same temperature. 


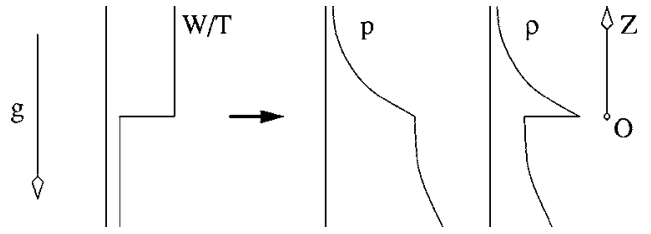

FIG. 2. Profiles of the thermodynamic variables in a system formed by two layers of homogeneous fluids, which is Rayleigh-Taylor unstable.

The pressure is obtained by substituting the expression for the density from the equation of state, Eq. (8), into the balance equation, Eq. (7), which yields

$$
p(z)=p_{0} \exp \left(-\frac{g W_{H}}{R^{0} T_{H}} \int_{0}^{z} \frac{W(\zeta) / W_{H}}{T(\zeta) / T_{H}} d \zeta\right),
$$

$p_{0}$ being the value of the pressure at the middle plane, $z=0$. This equation shows that the pressure decays exponentially with increasing height with a characteristic length scale

$$
L_{H}=\frac{R^{0} T_{H}}{g W_{H}},
$$

the scale-height, ${ }^{32}$ which is directly related to the speed of sound, in particular, proportional to $c_{H}^{2} / g$. There is a similar length scale associated with the light fluid

$$
L_{L}=\frac{R^{0} T_{L}}{g W_{L}} .
$$

The density profile is then computed from the equation of state, which yields

$$
\rho(z)=\rho_{0}^{+} \frac{W(z) / W_{H}}{T(z) / T_{H}} \exp \left(-\frac{1}{L_{H}} \int_{0}^{z} \frac{W(\zeta) / W_{H}}{T(\zeta) / T_{H}} d \zeta\right),
$$

$\rho_{0}^{+}$being related to $p_{0}$ by

$$
\rho_{0}^{+}=\frac{p_{0} W_{H}}{R^{0} T_{H}}=\frac{p_{0}}{g L_{H}} .
$$

Either $\rho_{0}^{+}$or $p_{0}$ has to be provided. It is interesting to note that they are also linked to the amount of mass deposited on the top, $m_{H}$, a relation obtained by integrating the density profile along the $O Z$ axis. Sketches of the different profiles are presented in Fig. 2.

The density has a jump at the interface $z=0$ from $\rho_{0}^{-}$at $z=0^{-}$to $\rho_{0}^{+}$at $z=0^{+}$given by

$$
\frac{\rho_{0}^{+}}{\rho_{0}^{-}}=\frac{W_{H} T_{L}}{W_{L} T_{H}}=\frac{L_{L}}{L_{H}},
$$

where the last equality follows from the definition of the two scale-heights. This equation shows that, if $\rho_{0}^{+} / \rho_{0}^{-}>1$, a condition required to have Rayleigh-Taylor instability, then $T_{H} / W_{H}<T_{L} / W_{L}$ and, consequently, $L_{H}<L_{L}$.

\section{Energy analysis}

In order to estimate the characteristic intensity of turbulent fluctuations $q_{0}$, the available potential energy of the system is calculated, since this constitutes an upper limit to the kinetic energy released to the flow. The length over which the profile jumps from $W_{L} / T_{L}$ to $W_{H} / T_{H}$ is assumed to be small compared to the scale-heights, the latter being defined by Eqs. (12) and (13). The density profile is

$$
\rho(z)= \begin{cases}\rho_{0}^{+} e^{-z / L_{H}}, & z>0 \\ \rho_{0}^{-} e^{-z / L_{L}}, & z<0\end{cases}
$$

according to Eq. (14).

A mass of light fluid $m_{L}$ is deposited over a domain depth $D_{L}$ and, on top of it, a mass of heavy fluid $m_{H}$ over a depth $D_{H}$. These four lengths and two masses completely define the system, since

$$
\begin{aligned}
& m_{H}=\int_{0}^{D_{H}} \rho(z) d z=\rho_{0}^{+} L_{H}\left(1-e^{-D_{H} / L_{H}}\right), \\
& m_{L}=\int_{-D_{L}}^{0} \rho(z) d z=\rho_{0}^{-} L_{L}\left(e^{D_{L} / L_{L}}-1\right)
\end{aligned}
$$

can be used to obtain $\rho_{0}^{+}$and $\rho_{0}^{-}$. The equation of state provides the pressure, if desired.

This system is not in stable equilibrium, and, either slowly by diffusion or more rapidly by Rayleigh-Taylor instability, it evolves toward a totally mixed configuration. A final buoyancy-stable configuration with $T_{F} / W_{F}$ constant is assumed. The final density distribution is

$$
\rho_{f}(z)=\rho_{F} e^{-\left(z+D_{L}\right) / L_{F}},
$$

where the density at the bottom, $\rho_{F}$, is the final density at $z$ $=-D_{L}$ and the scale-height of the final state is defined by

$$
L_{F}=\frac{R^{0} T_{F}}{g W_{F}} .
$$

The constants $\rho_{F}$ and $L_{F}$ need to be determined. One equation is the conservation of total mass

$$
m_{H}+m_{L}=\rho_{F} L_{F}\left(1-e^{\left.-\left(D_{L}+D_{H}\right) / L_{F}\right)} .\right.
$$

A second equation is derived from the conservation of total mass of each component separately. Working with the heavy fluid, its mass fraction is given, in general, by

$$
Y_{H}(z)=\frac{1-W_{L} / W(z)}{1-W_{L} / W_{H}},
$$

and the mass of heavy fluid is the integral of the profile $\rho(z) Y_{H}(z)$ between $-D_{L}$ and $D_{H}$, which yields the relation

$$
\frac{m_{H}}{m_{H}+m_{L}}=\frac{1-\psi}{1-W_{L} / W_{H}},
$$

with

$$
\psi=\frac{W_{L}}{m_{H}+m_{L}} \int_{-D_{L}}^{D_{H}} \frac{\rho}{W} d z .
$$

Conservation of the mass of heavy fluid, $m_{H}$, implies then that the function $\psi$ is equal between the initial and the final states. For the initial configuration, which is buoyancy-stable and isothermal in each of the two layers, it is 


$$
\psi_{1, i}=\frac{\left(e^{D_{L} / L_{L}}-1\right)+\left(1-e^{-D_{H} / L_{H}}\right) \frac{T_{L} L_{H}}{T_{H} L_{L}}}{e^{D_{L} / L_{L}}-e^{-D_{H} / L_{H}}},
$$

and for the final distribution

$$
\psi_{1, f}=\frac{W_{L}}{W_{F}} .
$$

Thus, the conservation of mass of the heavy species provides the equation

$$
\frac{W_{L}}{W_{F}}=\psi_{1, i},
$$

and from the definitions of the scales heights, Eqs. (13) and (20), we have

$$
\frac{L_{F}}{L_{L}}=\frac{W_{L} T_{F}}{W_{F} T_{L}}=\frac{T_{F}}{T_{L}} \psi_{1, i} .
$$

Equations (21) and (28) provide $\rho_{F} / \rho_{0}^{+}$, and $L_{F} / L_{L}$ as a funcion of $D_{H} / L_{L}, D_{L} / L_{L}$, and $L_{H} / L_{L}$, once $T_{F} / T_{L}$ and $T_{H} / T_{L}$ are known.

The potential energy of the fluid particle located at $z$ is given by $\rho(z) g z$, the reference level chosen at $z=0$, and the total amount of potential energy $V$ of a certain region in space is the integral of $\rho(z) g z$ over that region.

Hence, for the heavy fluid layer of the initial configuration we have

$$
\begin{aligned}
V_{H} & =\rho_{0}^{+} g L_{H}^{2}\left[1-e^{-D_{H} / L_{H}}\left(1+D_{H} / L_{H}\right)\right] \\
& =m_{H} g L_{H}-\rho_{0}^{+} g L_{H} D_{H} e^{-D_{H} / L_{H}} .
\end{aligned}
$$

The light fluid region yields

$$
\begin{aligned}
V_{L} & =\rho_{0}^{-} g L_{L}^{2}\left[e^{+D_{L} / L_{L}}\left(1-D_{L} / L_{L}\right)-1\right] \\
& =m_{L} g L_{L}-\rho_{0}^{-} g L_{L} D_{L} e^{D_{L} / L_{L}} .
\end{aligned}
$$

Lastly, the final configuration provides

$$
\begin{aligned}
V_{F} & =\rho_{F} g L_{F}^{2} e^{-D_{L} / L_{F}}\left[e^{+D_{L} / L_{F}}\left(1-D_{L} / L_{F}\right)-e^{-D_{H} / L_{F}}\left(1+D_{H} / L_{F}\right)\right] \\
& =\left(m_{H}+m_{L}\right) g L_{F}-\rho_{F} g L_{F}\left(D_{L}+D_{H} e^{-\left(D_{H}+D_{L} / L_{F}\right.}\right)
\end{aligned}
$$

The difference between the total initial potential energy and the final one is the available potential energy,

$$
E_{p}=V_{H}+V_{L}-V_{F}=E_{p, t}+E_{p, i},
$$

which has been decomposed as the sum of two parts

$$
\begin{aligned}
E_{p, t}= & \left(m_{H} g L_{H}+m_{L} g L_{L}\right)-\left(m_{H}+m_{L}\right) g L_{F}, \\
E_{p, i}= & \rho_{F} g L_{F}\left(D_{L}+D_{H} e^{-\left(D_{H}+D_{L}\right) / L_{F}}\right)-\rho_{0}^{+} g L_{H}\left(D_{L} e^{+D_{L} / L_{L}}\right. \\
& \left.-D_{H} e^{-D_{H} / L_{H}}\right) .
\end{aligned}
$$

It is the specific (per unit mass) available potential energy

$$
\frac{E_{p}}{\left(m_{H}+m_{L}\right)}
$$

which determines the velocity attained by the fluid.

Equations (25) and (28), under the assumption $T_{H}=T_{L}$, allow to write

$$
\frac{E_{p, t}}{\left(m_{H}+m_{L}\right) g L_{L}}=\psi_{i, 1}-\frac{L_{F}}{L_{L}}=\left(1-\frac{T_{F}}{T_{L}}\right) \psi_{i, 1},
$$

where $g L_{L}$ is used to normalize the specific potential energy. This is a thermal contribution to the change in potential energy, nonzero only in the case of change in temperature between the initial and the final states.

The second part, which is nonzero even for an isothermal case, can be written as

$$
\frac{E_{p, i}}{\left(m_{H}+m_{L}\right) g L_{L}}=\phi_{1}\left(\frac{D_{L}}{L_{L}}, \frac{D_{H}}{L_{L}}, \frac{L_{H}}{L_{L}}\right),
$$

where the function $\phi_{1}$ is defined by

$$
\begin{aligned}
\phi_{1}= & \frac{\left(D_{L} / L_{L}\right) e^{D_{L} / L_{F}}+\left(D_{H} / L_{L}\right) e^{-D_{H} / L_{F}}}{e^{D_{L} / L_{F}}-e^{-D_{H} / L_{F}}} \\
& -\frac{\left(D_{L} / L_{L}\right) e^{D_{L} / L_{L}}+\left(D_{H} / L_{L}\right) e^{-D_{H} / L_{H}}}{e^{D_{L} / L_{L}}-e^{-D_{H} / L_{H}}},
\end{aligned}
$$

and $L_{F} / L_{L}$ is given by Eq. (28) in terms of the length ratios of the problem shown as the arguments of $\phi_{1}$. The characteristic intensity of the turbulent fluctuations is given by $q_{0} / \sqrt{g L_{L}}=\sqrt{2 \phi_{1}}$.

The characteristic speed of sound $c_{0}$ is required at this stage in order to construct a characteristic turbulent Mach number. The speed of sound in the final mixed configuration (miscible fluids are being considered), $\gamma R^{0} T_{F} / W_{F}$, is used for this purpose, which normalized by $g L_{L}$ provides

$$
\frac{c_{0}^{2}}{g L_{L}}=\gamma \frac{L_{F}}{L_{L}}
$$

according to the definition of the final scale-height, Eq. (20). Hence, a characteristic turbulent Mach number is given by

$$
M_{t, 0}=\sqrt{\frac{2 \phi_{1}}{\gamma\left(L_{F} / L_{L}\right)}},
$$

which is a function of $D_{L} / L_{L}, D_{H} / L_{L}$, and $L_{H} / L_{L}$.

The mixing region $\left(-D_{L}, D_{H}\right)$ must now be estimated. A first estimate in the turbulent case for the upper limit of the final mixing region is given by the distance from the initial density jump until the point in the upper layer where the density becomes equal to $\rho_{0}^{-}$. This reasoning yields

$$
D_{H}=L_{H} \ln \frac{L_{L}}{L_{H}} .
$$

Similarly, the turbulent motion can develop toward the lower layer at most until the downward position $z=-D_{L}$ at which the initial density profile equals the value $\rho_{0}^{+}$. This distance is given by

$$
D_{L}=L_{L} \ln \frac{L_{L}}{L_{H}} .
$$

However, bubbles expand as they rise, and this could imply larger values of $D_{H}$ and $D_{L}$ if turbulent mixing is not fast enough to eliminate the density gradients through molecular diffusion. We explore this other limit now. Consider pure fluid bubbles that rise through the upper layer without turbulent mixing. Close to the interface there are bubbles 
with density $\rho_{b}=\rho_{0}^{-}$surrounded by a fluid with density $\rho_{0}^{+}$. These bubbles expand as they rise because the surrounding pressure is lower, and their density diminishes. If this process is adiabatic, then $\rho_{b} \propto p^{1 / \gamma}$, a smaller rate of decrease with height than the surrounding density, which varies proportionally to the pressure. Therefore, at a certain height, the density of the rising bubble is no longer smaller than the surrounding density and the buoyancy force is zero. This height determines the upper limit of the mixing zone and is given by the equation

$$
\rho_{0}^{-}\left(\frac{p\left(D_{H}\right)}{p_{0}}\right)^{1 / \gamma_{H}}=\rho_{0}^{+}\left(\frac{\rho\left(D_{H}\right)}{\rho_{0}^{+}}\right),
$$

where the density and the pressure follow the known exponential decay given by Eq. (17). The solution is

$$
D_{H}=\frac{\gamma_{H}}{\gamma_{H}-1} L_{H} \ln \frac{L_{L}}{L_{H}} \text {. }
$$

Similar reasoning provides an estimate for $D_{L}$,

$$
D_{L}=\frac{\gamma_{L}}{\gamma_{L}-1} L_{L} \ln \frac{L_{L}}{L_{H}} .
$$

These two lengths are equal to those defined by Eqs. (40) and (41) multiplied by the same constant $\gamma /(\gamma-1)$, greater than one, when a mean value of $\gamma$ is considered. Therefore, we can consider the mixing zone given by $\left(-\alpha D_{L}, \alpha D_{H}\right)$, with $D_{L}$ and $D_{H}$ given by Eqs. (43) and (44), and this approach covers also the former estimate of the thickness of the mixing layer for an intermediate value of $\alpha$. The parameter $\alpha$ varies between 0 and 1 , increasing in time corresponding to the evolution of $h(t)$. It has to be noted that the function $\phi_{1}$ gives the maximum specific available potential energy that would be released if the instantaneous mixing thickness $h(t)$ would be held constant sufficiently long to allow complete mixing. The actual potential energy being released is smaller because the characteristic time for mixing inside the region is the same as the characteristic time to engulf new mass into the mixing zone, namely, the turbulent time scale determined by the turbulent kinetic energy and the rate of turbulent dissipation.

To conclude the analysis, the maximum of $M_{t, 0}$, Eq. (39), along the curves $D_{L} / L_{L}=\alpha\left[\gamma_{L} /\left(\gamma_{L}-1\right)\right] \ln \left(L_{L} / L_{H}\right)$ and $D_{H} / L_{L}=\alpha\left[\gamma_{H} /\left(\gamma_{H}-1\right)\right]\left(L_{H} / L_{L}\right) \ln \left(L_{L} / L_{H}\right)$ for all values of $\alpha$ in the range $0<\alpha<1$ has to be calculated. When a mean value of $\gamma$ between 1 and 5/3 is substituted in that equation, the characteristic turbulent Mach number is found to be bounded by 0.62 . Note that this value is a conservative estimate, because a considerable amount of the available potential energy (of the order of 50\% according to the data regarding the incompressible case) is dissipated and does not contribute to the turbulent kinetic energy.

\section{B. Two buoyancy-neutral layers}

This section considers two layers which are buoyancyneutral, i.e., a relation $\rho(p)$ in each of them such that the buoyancy frequency is zero. Equation (6) with $N=0$ implies $\rho \propto p^{1 / \gamma}$. Hence, the thermodynamic state is defined by the group $p / \rho^{\gamma}$ varying between two uniform values $\left(p / \rho^{\gamma}\right)_{L}$ and $\left(p / \rho^{\gamma}\right)_{H}$, the profile $W / T$ being as required by the equation of state in order to obtain such a distribution. When the layers are pure fluids, then the entropy is constant inside each layer. This initial condition has been studied in the past by Chen et al. ${ }^{33}$ although they analyzed the two-dimensional problem with immiscible fluids, fundamentally different from the fully turbulent configuration with miscible fluids considered here.

For notational convenience, the profile $p^{1 / \gamma} / \rho$ is denoted by $s(z)$, which then varies from $s_{L}$ at the bottom to $s_{H}$ at the top. Substituting $\rho=p^{1 / \gamma} / s$ into the balance equation, Eq. (7), the pressure distribution is

$$
p(z)=p_{0}\left(1-\frac{\gamma-1}{\gamma} \frac{g}{p_{0}^{(\gamma-1) / \gamma} s_{H}} \int_{0}^{z} \frac{d \zeta}{s(\zeta) / s_{H}}\right)^{\gamma /(\gamma-1)},
$$

where $p_{0}$ is again the pressure at the center plane. The ratio of specific heats $\gamma$ has been taken as constant. The characteristic length scale is

$$
L_{H}=\frac{p_{0}^{(\gamma-1) / \gamma} S_{H}}{g}=\frac{p_{0}}{g \rho_{0}^{+}}=\frac{R^{0} T_{0}^{+}}{W_{0}^{+} g},
$$

having defined $\rho_{0}^{+}=p_{0}^{1 / \gamma} / s_{H}$. Similarly, the scale-height of the lower layer is

$$
L_{L}=\frac{p_{0}^{(\gamma-1) / \gamma} s_{L}}{g}=\frac{p_{0}}{g \rho_{0}^{-}}=\frac{R^{0} T_{0}^{-}}{W_{0}^{-} g} .
$$

The density profile is

$$
\rho(z)=\rho_{0}^{+} \frac{1}{s(z) / s_{H}}\left(1-\frac{\gamma-1}{\gamma} \frac{1}{L_{H}} \int_{0}^{z} \frac{d \zeta}{s(\zeta) / s_{H}}\right)^{1 /(\gamma-1)} .
$$

The density has a jump at the interface $z=0$ from $\rho_{0}^{-}$at $z$ $=0^{-}$to $\rho_{0}^{+}$at $z=0^{+}$given by

$$
\frac{\rho_{0}^{+}}{\rho_{0}^{-}}=\frac{s_{L}}{s_{H}}=\frac{L_{L}}{L_{H}} .
$$

It can be observed that the derivation is parallel to that of Sec. II A. In fact, that section considers $\rho \propto p$, which is the limit $\gamma \rightarrow 1$ of the case considered here, $\rho \propto p^{1 / \gamma}$. Since $\gamma$ is always of order one, this convergence suggests that there is no qualitative change in the bounding of $M_{t}$ with respect to the buoyancy-stable configuration presented in Sec. II A.

It is also observed that the density and the pressure fall down to zero along the upper layer on a distance of order $O\left[\gamma /(\gamma-1) L_{H}\right]$.

\section{Energy analysis}

The calculation of specific potential energy is now presented, neglecting once more the thickness of the initial interface with respect to the scale heights $L_{H}$ and $L_{L}$, Eqs. (46) and (47), respectively. The initial mean density profile is 


$$
\rho(z)=\left\{\begin{array}{l}
\rho_{0}^{+}\left(1-\frac{\gamma_{H}-1}{\gamma_{H}} \frac{z}{L_{H}}\right)^{1 /\left(\gamma_{H^{-1}}\right)}, \quad z>0 \\
\rho_{0}^{-}\left(1-\frac{\gamma_{L}-1}{\gamma_{L}} \frac{z}{L_{L}}\right)^{1 /\left(\gamma_{L}-1\right)}, \quad z<0
\end{array}\right.
$$

It can be verified that Eq. (50) tends toward Eq. (17) in the limit $\gamma \rightarrow 1$.

In a fashion similar to that of the previous sections, a mass of light mixed fluid $m_{L}$ is deposited over a domain depth $D_{L}$,

$$
m_{L}=\int_{-D_{L}}^{0} \rho(z) d z=\rho_{0}^{-} L_{L}\left[\left(1+\frac{\gamma_{L}-1}{\gamma_{L}} \frac{D_{L}}{L_{L}}\right)^{\gamma_{L} /\left(\gamma_{L^{-}}-1\right)}-1\right],
$$

and, on top of it, a mass of heavy mixed fluid $m_{H}$ over a depth $D_{H}$,

$$
m_{H}=\int_{0}^{D_{H}} \rho(z) d z=\rho_{0}^{+} L_{H}\left[1-\left(1-\frac{\gamma_{H}-1}{\gamma_{H}} \frac{D_{H}}{L_{H}}\right)^{\gamma_{H}\left(\gamma_{H^{-1}}\right)}\right] .
$$

The temperature is supposed to be uniform inside each layer, as done by Chen $e t$ al. ${ }^{33}$ and as assumed in Sec. II A as well. This condition implies that the two layers are not composed of pure fluid, but that the molecular weight varies, according to the equation of state, as

$$
\frac{1}{W(z)}= \begin{cases}\frac{1}{W_{0}^{+}}\left(1-\frac{\gamma_{H}-1}{\gamma_{H}} \frac{z}{L_{H}}\right), & z>0 \\ \frac{1}{W_{0}^{-}}\left(1-\frac{\gamma_{L}-1}{\gamma_{L}} \frac{z}{L_{L}}\right), & z<0 .\end{cases}
$$

The same reasoning as in the preceding section is now applied. The final state is considered to be a complete homogeneous mixture, buoyancy-stable, which provides the minimum final potential energy and therefore a conservative estimate for the potential energy released into the flow. The density is given by Eq. (19) and the conservation of total mass is expressed again by Eq. (21). The corresponding function $\psi_{2, i}$, defined by Eq. (24), is given by

$$
\frac{W_{0}}{W_{L}} \psi_{2, i}=\frac{\frac{\gamma_{L}}{2 \gamma_{L}-1}\left[\left(1+\frac{\gamma_{L}-1}{\gamma_{L}} \frac{D_{L}}{L_{L}}\right)^{\left(2 \gamma_{L}-1\right) /\left(\gamma_{L}-1\right)}-1\right]+\frac{\gamma_{H}}{2 \gamma_{H}-1}\left[1-\left(1-\frac{\gamma_{H}-1}{\gamma_{H}} \frac{D_{H}}{L_{H}}\right)^{\left(2 \gamma_{H^{-1}}\right) /\left(\gamma_{H^{-1}}\right)}\right] \frac{L_{H} T_{L}}{L_{L} T_{H}}}{\left(1+\frac{\gamma_{L}-1}{\gamma_{L}} \frac{D_{L}}{L_{L}}\right)^{\gamma_{L} /\left(\gamma_{L}-1\right)}-\left(1-\frac{\gamma_{H}-1}{\gamma_{H}} \frac{D_{H}}{L_{H}}\right)^{\gamma_{H} /\left(\gamma_{H^{-1}}\right)}} .
$$

It can be again verified that Eq. (54) recovers Eq. (25) in the limit $\gamma \rightarrow 1$. Then the conservation of the mass of heavy fluid implies, using Eq. (26),

$$
\frac{W_{L}}{W_{F}}=\psi_{2, i} \text {. }
$$

The final scale-height is given by Eq. (20), which can be written explicitly as

$$
\frac{L_{F}}{L_{L}}=\frac{T_{F}}{T_{L}} \frac{W_{0}}{W_{F}}=\frac{T_{F}}{T_{L}} \frac{W_{0}}{W_{L}} \psi_{2, i}
$$

with Eq. (47).
Calculating the potential energies as explained in Sec. II A $\left(T_{H}=T_{L}\right.$ is again assumed), the normalized specific available potential energy due to temperature changes becomes

$$
\frac{E_{p, t}}{\left(m_{H}+m_{L}\right) g L_{L}}=\frac{W_{0}^{-}}{W_{L}} \psi_{2, i}-\frac{L_{F}}{L_{L}}=\left(1-\frac{T_{F}}{T_{L}}\right) \frac{W_{0}^{-}}{W_{L}} \psi_{2, i} .
$$

The remaining part is written as

$$
\frac{E_{p, i}}{\left(m_{H}+m_{L}\right) g L_{L}}=\phi_{2}\left(\frac{D_{L}}{L_{L}}, \frac{D_{H}}{L_{L}}, \frac{L_{H}}{L_{L}}\right),
$$

where $\phi_{2}$ is defined by

$$
\phi_{2}=\frac{\left(D_{L} / L_{L}\right) e^{D_{L} / L_{F}}+\left(D_{H} / L_{L}\right) e^{-D_{H} / L_{F}}}{e^{D_{L} / L_{F}}-e^{-D_{H} / L_{F}}}-\frac{\frac{D_{L}}{L_{L}}\left(1+\frac{\gamma_{L}-1}{\gamma_{L}} \frac{D_{L}}{L_{L}}\right)^{\gamma_{L} /\left(\gamma_{L}-1\right)}+\frac{D_{H}}{L_{L}}\left(1-\frac{\gamma_{H}-1}{\gamma_{H}} \frac{D_{H}}{L_{H}}\right)^{\gamma_{H} /\left(\gamma_{H^{-1}}\right)}}{\left(1+\frac{\gamma_{L}-1}{\gamma_{L}} \frac{D_{L}}{L_{L}}\right)^{\gamma_{L} /\left(\gamma_{L}-1\right)}-\left(1-\frac{\gamma_{H}-1}{\gamma_{H}} \frac{D_{H}}{L_{H}}\right)^{\gamma_{H} /\left(\gamma_{H^{-1}}\right)}} .
$$


The characteristic intensity of the turbulent fluctuations is given by $q_{0} / \sqrt{g L_{L}}=\sqrt{2 \phi_{2}}$.

The characteristic speed of sound $c_{0}$ is now needed. The squared speed of sound in the final mixed configuration is $\gamma R^{0} T_{F} / W_{F}$, which normalized by $g L_{L}$ provides

$$
\frac{c_{0}^{2}}{g L_{L}}=\gamma \frac{L_{F}}{L_{L}}
$$

according to the definition of the final scale-height, Eq. (20). Finally, a characteristic turbulent Mach number is given by

$$
M_{t, 0}=\sqrt{\frac{2 \phi_{2}}{\gamma\left(L_{F} / L_{L}\right)}},
$$

similar to Eq. (39) but with the corresponding function $\phi_{2}$.

The final mixing region $\left(-D_{L}, D_{H}\right)$ has to be estimated. A first estimate for the upper limit of the final mixing region is given by the distance from the initial density jump until the point in the upper layer where the density becomes equal to $\overline{\rho_{0}^{-}}$. This reasoning yields

$$
D_{H}=\frac{\gamma_{H}}{\gamma_{H}-1} L_{H}\left[1-\left(\frac{L_{H}}{L_{L}}\right)^{\gamma_{H^{-1}}}\right] \text {. }
$$

Similarly, the turbulent motion can develop toward the lower layer until the downward position $z=-D_{L}$ at which the initial density profile equals the value $\rho_{0}^{+}$. This distance is given by

$$
D_{L}=\frac{\gamma_{L}}{\gamma_{L}-1} L_{L}\left[\left(\frac{L_{L}}{L_{H}}\right)^{\gamma_{L}-1}-1\right] \text {. }
$$

Now, the role of the neutral stability of each initial layer has to be considered, as it was done in Sec. II A. The same reasoning here implies that pure bubbles continually rise through all the available upper layer, whose limit is given by the point where the density becomes zero. From Eq. (50), this singularity occurs at a distance

$$
D_{H}=L_{H} \frac{\gamma_{H}}{\gamma_{H}-1} \text {. }
$$

Therefore, we consider the mixing zone given by $\left(-\alpha D_{L}, \alpha D_{H}\right)$, with $\alpha$ varying between 0 and $1, D_{H}$ given by Eq. (64), and

$$
D_{L}=D_{H}\left(\frac{L_{L}}{L_{H}}\right)^{\gamma} \text {. }
$$

Such a choice for the mixing zone covers that described by Eqs. (62) and (63) for some intermediate value of $\alpha$, and includes the whole upper layer when $\alpha=1$. Note that Eq. (65) recovers the ratio $D_{L} / D_{H}$ of the buoyancy-stable initial configuration, Sec. II A, in the limit $\gamma \rightarrow 1$.

The maximum of $M_{t, 0}$, Eq. (61), along the previous curves $D_{L} / L_{L}=\alpha[\gamma /(\gamma-1)]\left(L_{L} / L_{H}\right)^{(\gamma-1)}$ and $D_{H} / L_{L}$ $=\alpha[\gamma /(\gamma-1)]\left(L_{H} / L_{L}\right)$ for $0<\alpha<1$, is now to be calculated. When a mean value of $\gamma$ between 1 and 5/3 is substituted in this equation, the characteristic turbulent Mach number is bounded by 0.57 . The reader is again reminded that all the potential energy is assumed to be converted to turbulent kinetic energy without any loss to molecular dissipation; therefore, this upper bound of $M_{t, 0}$ is a conservative value.

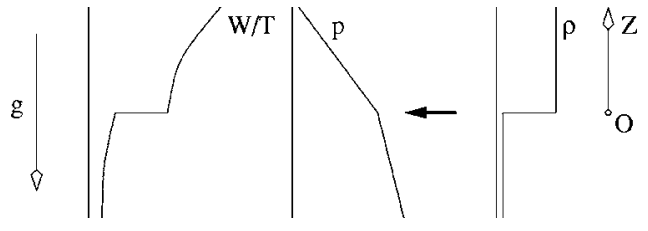

FIG. 3. Profiles of the thermodynamic variables in a system formed by two layers of constant density, which is Rayleigh-Taylor unstable.

\section{Two buoyancy-unstable layers}

This section considers two layers which are buoyancyunstable, i.e., a relation $\rho(p)$ in each layer such that the buoyancy-frequency is negative. The particular configuration, shown in Fig. 3, is formed by two layers of constant density with a jump from $\rho_{L}$ to $\rho_{H}$ (larger) at the interface $z=0$, the profile $T / W$ varying as required by the equation of state in order to obtain such a distribution. Equation (6) with $\rho$ constant implies $N^{2}<0$ inside each layer. This case might not be set up as easily as the one considered in the previous sections (it is unstable), and it will be not considered with such generality, but it is of interest for several reasons. First, because it represents a hydrostatic profile that is unstable. Second, the Atwood number based on the mean density profile is constant instead of decreasing with time (although the effective Atwood number increases, as it is later shown in the simulations). Third, there are data available in the literature for the incompressible limit of this configuration that can be utilized to validate the LES results.

Given $\rho(z)$, the integration of Eq. (7) yields

$$
p(z)=p_{0}\left(1-\frac{\rho_{H} g}{p_{0}} \int_{0}^{z} \frac{\rho(\zeta)}{\rho_{H}} d \zeta\right) .
$$

The scales that characterize the thermodynamic variation are

$$
L_{H}=\frac{p_{0}}{\rho_{H} g},
$$

similar to Eq. (15) with $\rho_{0}^{+}$in that equation corresponding to $\rho_{H}$ in Eq. (67), and

$$
L_{L}=\frac{p_{0}}{\rho_{L} g} .
$$

The required ratio $T(z) / W(z)$ is calculated from the equation of state,

$$
\frac{T(z)}{W(z)}=\left(\frac{T}{W}\right)_{0}^{+} \frac{1-\int_{0}^{z} \rho(\zeta) / \rho_{H} d \zeta / L_{H}}{\rho(z) / \rho_{H}},
$$

where

$$
\left(\frac{T}{W}\right)_{0}^{+}=\frac{p_{0}}{R^{0} \rho_{H}} .
$$

The profiles are sketched in Fig. 3. The constancy of density over each (heavy or light) fluid column can be achieved by either decreasing the temperature or increasing the molecular weight as $z$ increases. Needless to say, the distribution $W(z)$ 
determines the two species to be used in the problem, since the relation

$$
W_{L}<W(z)<W_{H}
$$

holds.

In this configuration, the buoyancy frequency, Eq. (6), is negative, and this instability implies that the whole domain will be involved in the mixing process. However, it is immediately observed from the linear decrease in the pressure that the maximum domain length cannot be larger than $O\left(L_{H}\right)$, given by Eq. (67), because the pressure reaches zero at that limit (we would need either zero absolute temperature or a species of infinite molecular weight). The problem is studied up to this instant of time.

\section{Energy analysis}

An analysis based on the specific available potential energy similar to that of the preceding section is performed again. The thickness of the density jump is neglected and the density profile is

$$
\rho(z)= \begin{cases}\rho_{H}, & z>0 \\ \rho_{L}, & z<0 .\end{cases}
$$

We consider a mixing region between $-D_{L}$ and $D_{H}$. Based on the available incompressible data, ${ }^{13,15}$ the density profiles at each time are estimated approximately by a linear variation from $\rho_{L}$ at $z=-D_{L}$ to $\rho_{H}$ at $z=+D_{H}$. This assumption is later verified in the simulation for the compressible case. Conservation of mass inside this mixing region then implies $D_{H}=D_{L}$. Experimental results show, however, that spikes evolve significantly faster than bubbles when the density ratio is large enough, ${ }^{8}$ therefore, the analysis presented here for this configuration is applicable only to relatively small Atwood numbers, $A \lesssim 0.5$.

Utilizing the same formulation as in the preceding section, the potential energies are

$$
\begin{aligned}
& V_{H}=\rho_{H} g D_{H}^{2} / 2, \\
& V_{L}=-\rho_{L} g D_{H}^{2} / 2, \\
& V_{F}=\left(\rho_{H}-\rho_{L}\right) g D_{H}^{2} / 3,
\end{aligned}
$$

the available potential energy is $E_{p}=V_{H}+V_{L}-V_{F}$, and the normalized specific available potential energy is

$$
\frac{E_{p}}{\left(m_{H}+m_{L}\right) g L_{L}}=\phi_{3}\left(\frac{D_{H}}{L_{L}}, \frac{L_{H}}{L_{L}}\right)
$$

where

$$
\phi_{3}=\frac{1}{6} A \frac{D_{H}}{L_{L}}=\frac{1}{6} \frac{L_{L} / L_{H}-1}{L_{L} / L_{H}+1} \frac{D_{H}}{L_{L}} .
$$

A characteristic turbulent intensity is then given by $q_{0} / \sqrt{g L_{L}}=\sqrt{2 \phi_{3}}$.

On the other hand, an estimate of the characteristic speed of sound at the center plane, $c_{0}$, can be obtained now using a mean value $\left(p_{0} / \rho_{H}+p_{0} / \rho_{L}\right) / 2$ in Eq. (9) and the definitions Eqs. (67) and (68), which yields

$$
\frac{c_{0}^{2}}{g L_{L}}=\frac{\gamma}{2}\left(1+\frac{L_{H}}{L_{L}}\right)
$$

The minimum of the speed of sound corresponds to the upper limit of the domain, where the pressure is minimum, and it can be made as small as desired by reducing $p_{0}$. However, the turbulent motion develops around the middle plane and it is the value of $c$ there that matters. Hence, a characteristic turbulent Mach number is given by

$$
M_{t, 0}=\sqrt{\frac{4 \phi_{3}}{\gamma\left(1+L_{H} / L_{L}\right)}},
$$

which is a function of $D_{H} / L_{L}$ and $L_{H} / L_{L}$, the function $\phi_{3}$ defined by Eq. (75).

The final size of the mixing region $D_{H} / L_{L}$ must now be expressed in terms of the given configuration $L_{H} / L_{L}$ $=\rho_{L} / \rho_{H}$. As already mentioned before, the upper domain height $D_{H}$ cannot exceed $L_{H}$ so that the maximum value that the mixing zone can reach is precisely

$$
\frac{D_{H}}{L_{L}}=\frac{L_{H}}{L_{L}}
$$

The maximum of the function $M_{t, 0}$, defined in Eq. (77), along the curve $D_{H} / L_{L}=f\left(L_{H} / L_{L}\right)$ gives an upper bound for the turbulent Mach number of 0.25 , having used again a mean value of $\gamma$ between 1 and 5/3. Note that $\phi_{3}$, Eq. (75), increases monotonically with the mixing region $D_{H} / L_{L}$, and the final value of $D_{H} / L_{L}$ used above maximizes the intermediate states $\alpha D_{H} / L_{L}, 0<\alpha<1$.

\section{Summary of the analytical results}

The major result of Sec. II is that the turbulent Mach number has an upper bound, independent of the density ratio under a wide range of initial thermodynamic configurations, which may not be large enough for intrinsic compressibility effects to be important in Rayleigh-Taylor turbulence. This result holds for three different choices of static stability inside each layer: stable, neutral, and unstable. An assumption underlying the analysis is that the flow is fully turbulent. In this respect, if a large scale perturbation, $O\left(L_{H}\right)$, is imposed initially at the interface then there could be compressibility effects as a blob of pure fluid rises/falls into the opposite pure fluid layer; this is not the case studied here. Also, miscible fluids subject to a turbulent flow have been considered, which allows the characteristic speed of sound to be estimated by that of the mixed fluid. This aspect of the problem could be different in the case of immiscible fluids. Another assumption is that of an ideal gas. The fundamental cause of the limitation of $M_{t}$ is independent of this latter assumption, however, the particular upper bound of the turbulent Mach number found in the analysis depends on the details of each equation of state.

It is also worth noticing that Secs. II A and II B consider 
a final state that is buoyancy-stable. A final state that is buoyancy-neutral could also have been assumed. However, the latter yields higher final potential energy, i.e., smaller characteristic turbulent Mach number. Thus the former provides a conservative estimate.

The aforementioned work of Chen et al..$^{33}$ reaches a different conclusion, reporting a significant influence of compressibility on the development of the flow. However, this reference considers immiscible fluids and it is twodimensional, which is fundamentally different from the case addressed here, namely, miscible fluids and a threedimensional configuration. In the two-dimensional case, larger and larger bubbles appear without a transfer of energy toward smaller scales. Furthermore, there is no molecular mixing and therefore the effective Atwood number cannot be reduced with respect to the completely segregated state, this result being radically different from what is observed in the miscible three-dimensional case, ${ }^{15}$ where the effective Atwood number can be half of the immiscible one. Hence, in the two-dimensional case, larger and fewer bubbles of immiscible fluid are present and the only possibility for the effective Atwood number is to increase due to the expansion as they rise. In the three-dimensional problem, however, this expansion due to compressibility is counteracted by mixing, and precisely what our investigation shows is that turbulent mixing dominates over this expansion phenomenon in the case of a fully turbulent flow. Therefore, the results obtained here do not contradict Chen et al. $;^{33}$ we simply look at a different problem.

\section{FORMULATION OF THE NUMERICAL SIMULATIONS}

\section{A. Governing equations and subgrid model}

A spatial filter operation over a field $\phi(\mathbf{x})$ representing any physical property per unit volume (pressure or density) at a fixed time is defined by

$$
\bar{\phi}(\mathbf{x})=\int_{\Omega} \phi(\mathbf{r}) G(\mathbf{x}-\mathbf{r}) d \mathbf{r},
$$

where the filter kernel $G(\mathbf{x}, \mathbf{r})$ has been assumed to be homogeneous in space and the integral extends over the flow domain. The filtered field of any quantity per unit mass $\psi(\mathbf{x})$ (velocity, species mass fraction, or the group $R^{0} T / W$ ), is Favre or density weighted,

$$
\bar{\psi}(\mathbf{x})=\int_{\Omega} \rho(\mathbf{r}) \psi(\mathbf{r}) G(\mathbf{x}-\mathbf{r}) d \mathbf{r} / \int_{\Omega} \rho(\mathbf{r}) G(\mathbf{x}-\mathbf{r}) d \mathbf{r},
$$

yet it will be denoted equally by an overbar in order to simplify the notation of the subgrid-scale model.

The equations used to describe the flow are obtained by applying this filter to the Navier-Stokes equations, which, after certain simplifying assumptions described below, yield the system

$$
\begin{aligned}
& \frac{\partial \bar{\rho}}{\partial t}+\nabla \cdot(\bar{\rho} \overline{\mathbf{u}})=0, \\
& \frac{\partial(\bar{\rho} \overline{\mathbf{u}})}{\partial t}+\boldsymbol{\nabla} \cdot(\bar{\rho} \overline{\mathbf{u}} \overline{\mathbf{u}})=-\nabla \bar{p}-\boldsymbol{\nabla} \cdot \mathbf{q}_{u}+\bar{\rho} \mathbf{g}, \\
& \frac{\partial \bar{p}}{\partial t}+\overline{\mathbf{u}} \cdot \boldsymbol{\nabla} \bar{p}=-\gamma \bar{p} \boldsymbol{\nabla} \cdot \overline{\mathbf{u}}-\boldsymbol{\nabla} \cdot \mathbf{q}_{p}, \\
& \frac{\partial(\bar{\rho} \bar{Y})}{\partial t}+\boldsymbol{\nabla} \cdot(\bar{\rho} \overline{\mathbf{u}} \bar{Y})=-\boldsymbol{\nabla} \cdot \mathbf{q}_{Y} .
\end{aligned}
$$

Henceforth, $Y$ is the species mass fraction of heavy fluid $Y_{H}$, and the species mass fraction of the light fluid is given by $Y_{L}=1-Y$. The subgrid-scale terms are defined by

$$
\begin{aligned}
& \mathbf{q}_{u}=\bar{\rho}(\overline{\mathbf{u u}}-\overline{\mathbf{u}} \overline{\mathbf{u}}), \\
& \mathbf{q}_{p}=(\overline{p \mathbf{u}}-\bar{p} \overline{\mathbf{u}}), \\
& \mathbf{q}_{Y}=\bar{\rho}(\overline{\mathbf{u} Y}-\overline{\mathbf{u}} \bar{Y}) .
\end{aligned}
$$

The following simplifications have been adopted. First, the Reynolds number is assumed to be large enough for the molecular dissipation terms to be negligible compared to the subgrid-scale terms. The second simplification has been done in the pressure equation, where the subgrid-scale pressuredilatation term has been dropped. It has been documented that the average value of this term is negligible for low-tomoderate Mach numbers ${ }^{24}$ and further investigation ${ }^{34}$ has shown that this term has a negligible impact on the LES results. The ratio of specific heats $\gamma$ is given by

$$
\gamma=\frac{1}{1-R^{0} /\left[C_{p} W(Y)\right]},
$$

the resolved part of which is denoted by $\gamma$. The specific heat of the mixture at constant pressure $C_{p}$ has been taken as a constant. The subgrid-scale term arising from the product of $\gamma$ and the pressure-dilatation correlation has been neglected due to the small variation of the ratio of specific heats $(1 \leqslant \gamma \leqslant 5 / 3)$.

The filtered equation of state is written as

$$
\overline{\bar{p}}=R^{0} \bar{T}\left(\sum_{i=L, H} \frac{\bar{Y}_{i}}{W_{i}}+\sum_{i=L, H} \frac{\overline{T Y_{i}}-\bar{T} \bar{Y}_{i}}{W_{i}}\right) .
$$

Isothermal conditions are imposed, and the subgrid-scale term in the above equation arising from the temperature fluctuations is expected to be small compared to the resolved part.

The coupling between the species mass fractions and the flow variables enters the original (not filtered) equations mainly through the viscous terms. ${ }^{13}$ Since the highReynolds-number case is considered here, the only coupling enters through the ratio of specific heats $\gamma$, Eq. (83), and this coupling is expected to be small. Hence, the species mass fraction behaves basically as a passive scalar.

The subgrid-scale model employed to close the remaining subfilter terms in the governing equations is a dynamic 
mixed model. ${ }^{34}$ This model is able to allow backscatter and to adapt itself to the flow characteristics at each time, its main drawback being the high computational cost. It is shown $^{35}$ thorough a posteriori comparisons among the most usual closure approaches that the dynamic mixed model, at least for the case analyzed there (mixing layer), is superior (though not perfect) to the rest of the closures, and the review of Meneveau et al. ${ }^{18}$ also points out several advantages of this subgrid model. It has to be realized, however, that the present problem is characterized by strong variations of the density. The differences between the constant-density and the variable-density flows, from a LES point of view, are that, in the latter case, the energy equation has to be solved and the isotropic part of subgrid-scales shear stress has to be modeled (cannot be included in a modified pressure). Several studies have been made in this direction. ${ }^{34,36-40}$

Hence, the closure of the system, Eq. (81), is done by

$$
\begin{aligned}
& \mathbf{q}_{u}^{d} \simeq \overline{\bar{\rho}}(\overline{\overline{\mathbf{u}} \overline{\mathbf{u}}}-\overline{\overline{\mathbf{u}}} \overline{\overline{\mathbf{u}}})^{d}-2 \bar{\rho} \nu_{s g} \mathbf{S}_{f}^{d}, \\
& \mathbf{q}_{u}^{i} \simeq \overline{\bar{\rho}}(\overline{\overline{\mathbf{u}} \overline{\mathbf{u}}}-\overline{\overline{\mathbf{u}}} \overline{\overline{\mathbf{u}}})^{i}, \\
& \mathbf{q}_{p} \simeq(\overline{\overline{\mathbf{u}} \bar{p}}-\overline{\overline{\mathbf{u}}} \overline{\bar{p}})-\bar{\rho} D_{p, s g} \boldsymbol{\nabla}(\bar{p} / \bar{\rho}), \\
& \mathbf{q}_{Y} \simeq \overline{\bar{\rho}}(\overline{\overline{\mathbf{u}} \bar{Y}}-\overline{\overline{\mathbf{u}}} \overline{\bar{Y}})-\bar{\rho} D_{Y, s g} \boldsymbol{\nabla} \bar{Y},
\end{aligned}
$$

where $\nu_{s g}=C_{u} \Delta_{f}^{2}\left|\mathbf{S}_{f}\right|, D_{p, s g}=C_{p} \Delta_{f}^{2}\left|\mathbf{S}_{f}\right|$, and $D_{Y, s g}=C_{Y} \Delta_{f}^{2}\left|\mathbf{S}_{f}\right|$, and $C_{u}, C_{p}$, and $C_{Y}$ are computed dynamically. $\mathbf{S}_{f}^{d}$ is the deviatoric part of the strain rate tensor of the filtered velocity field $\overline{\mathbf{u}}$ and $\left|\mathbf{S}_{f}\right|$ is defined by $\left(2 S_{f, i j} S_{f, i j}\right)^{1 / 2}$.

The eddy-viscosity part of the model for the isotropic component of the subgrid-scale stress tensor is not retained. First, there is a negligible impact of this isotropic part on the flow, since it is small compared to the thermodynamic pressure for moderate Mach numbers. ${ }^{38,39}$ Besides, it has been reported that the Smagorinsky contribution to it may lead to instability of the LES (Ref. 34) and provides a lower correlation with exact values in the a priori analysis. ${ }^{34,37}$

The dynamic methodology is applied using the basic filter $(F$ level $)$ at $\Delta_{f}$ and the test filter $(G$ level $)$ at $\Delta_{f}$ as well. Application of these two filters defines a new filter $(F G$ level), with an associated filter scale $\kappa \Delta_{f}$. The value of $\kappa$, computed as suggested by Vreman et al., ${ }^{35}$ is $\sqrt{2}$.

\section{B. Numerical scheme}

The governing equations, written in Cartesian coordinates, are discretized on a structured uniform mesh with the same grid spacing in each direction. The spatial derivatives are computed using a compact Padé scheme of sixth-order accuracy. ${ }^{41}$ This numerical scheme yields numerical errors that are small compared to the subgrid-scale terms of the governing equations when the filter size is chosen as twice the grid size, ${ }^{42}$ as it is done here. It is biased inward at the nonperiodic boundaries (top and bottom), having locally third-order accuracy. The advancement in time is performed with a low-storage fourth-order Runge-Kutta scheme. ${ }^{43}$ The boundary conditions in the nonperiodic directions are implemented in characteristic nonreflective form. ${ }^{44}$
A compact filter ${ }^{41}$ is applied to remove the aliased energy at the high wavenumbers originated by the nonlinear interaction of resolved-scale modes. The diffusive terms arising from the subgrid-scale models are treated in conservative form, and, therefore, two successive first-order difference operators are used. The transfer function of this operator ${ }^{41}$ is characterized by falling to zero at the highest wavenumber, which eliminates any dissipation at those scales. The frequency and intensity of the filter is set in order to remove an amount of energy that is negligible compared to the subgrid dissipation. This ratio is monitored along the simulation and maintained of the order of $5 \%$ of the subgrid-scale dissipation.

The nonlinear terms are treated in the skew-symmetric form to reduce aliasing errors,

$$
\begin{aligned}
& \mathcal{N}_{u}=-\frac{1}{2}[\boldsymbol{\nabla} \cdot(\bar{\rho} \bar{u} \bar{u})+\bar{\rho} \bar{u} \cdot \boldsymbol{\nabla} \bar{u}+\bar{u} \boldsymbol{\nabla} \cdot(\bar{\rho} \bar{u})] \\
& \mathcal{N}_{Y}=-\frac{1}{2}[\boldsymbol{\nabla} \cdot(\bar{\rho} \bar{u} \bar{Y})+\bar{\rho} \bar{u} \cdot \boldsymbol{\nabla} \bar{Y}+\bar{Y} \boldsymbol{\nabla} \cdot(\bar{\rho} \bar{u})] .
\end{aligned}
$$

This formulation, along with the symmetric differencing scheme, ensures conservation of mass, momentum, and kinetic energy inside the domain and across periodic boundary conditions with respect to the nonlinear terms. ${ }^{39,45,46}$ It is noted that there are two slightly different skew-symmetric formulations for variable density flows. ${ }^{39,47}$ Two-dimensional simulations of the Navier-Stokes equations without filtering showed that the regrouping proposed by Erlebacher et al. ${ }^{39}$ was stable for a larger number of iterations, and, therefore, it was chosen for the present LES.

\section{SIMULATION OF THE CONFIGURATION WITH EACH LAYER AT CONSTANT COMPOSITION}

\section{A. Problem definition}

The configuration shown in Fig. 2 and analyzed in Sec. II A is numerically simulated. The species mass fraction has a smooth transition from 0 (bottom) to 1 (top) over a thickness $h_{Y, 0}$ and the temperature is constant everywhere. Initially, the system has no fluctuations. At time $t=0$, the interface between the two fluids is perturbed and the temporal development of the resultant Rayleigh-Taylor instability into turbulence is simulated.

The problem is nondimensionalized with $p_{0}, g$, and the thermodynamic state of the pure heavy fluid, $W_{H}$ and $T_{H}$. A length scale is given by $L_{H}$, Eq. (12), and velocities are nondimensionalized with $\sqrt{g L_{H}}$ and densities with $\rho_{0}^{+}$, defined by Eq. (15). The nondimensional governing equations for the nondimensional variables $\rho^{*}, \mathbf{u}^{*}, p^{*}$, and $Y$ take the same form as the original system, Eq. (81), with

$$
\gamma^{\prime}=\frac{\gamma_{H}}{\gamma_{H}-\left(\gamma_{H}-1\right) / W^{*}},
$$

where, from Eq. (83), the reference ratio of specific heats is $\gamma_{H}=1 /\left[1-R^{0} /\left(C_{p} W_{H}\right)\right]$. Hence, only $\gamma_{H}$ enters in the governing equations described in the preceding section. Henceforth, the asterisk denotes nondimensional values. The overbar denoting filtered quantities has been dropped for notational convenience. 
The initial condition for the species mass fraction is given by

$$
Y\left(z^{*}\right)=\frac{1}{2}\left[1+\operatorname{erf}\left(\frac{z^{*}+\xi^{*}(x, y)}{2 h_{Y, 0}^{*} / 6.58}\right)\right],
$$

varying between 0 at the bottom and 1 at the top. In this equation, $h_{Y, 0}^{*}$ is the initial thickness of the mixing layer and the initial perturbation is described by $\xi^{*}(x, y)$. The thickness $h_{y}^{*}$ is defined by the length over which the mean mass fraction profile varies from 0.01 to 0.99 . Note that the mixing thickness in this configuration is based on the mass fraction profiles and not on the density profile because the latter varies exponentially with the vertical distance from the center plane.

This distribution of species mass fractions imposes a variation of the nondimensional molecular weight as follows:

$$
\frac{1}{W^{*}}=Y+\frac{1-Y}{W_{L}^{*}} .
$$

The initial pressure is given by Eq. (11), which written in nondimensional variables reads

$$
p^{*}\left(z^{*}\right)=\exp \left(-\int_{0}^{z^{*}} W^{*}(\zeta) d \zeta\right) .
$$

The density is then calculated by $\rho^{*}=p^{*} W^{*} / T^{*}$, where $T^{*}$ $=1$ has been assumed in this case, as said before.

The nondimensional external parameters defining the isothermal problem are therefore $h_{Y, 0}^{*}=h_{Y, 0} / L_{H}, W_{L}^{*}$ $=W_{L} / W_{H}$, and $\gamma_{H}$. The effect of the molecular weight ratio on compressibility has already been analyzed in Sec. II. The influence of varying $\gamma_{H}$ is expected to be small because the ratio of specific heats varies between 5/3 and 1 in the case of an ideal gas.

The ratio of molecular weights is $W_{L}^{*}=1 / 3$ (initial density jump at the interface $\rho_{0}^{+} / \rho_{0}^{-}=3$, initial Atwood number $A=0.5)$. The reference ratio of specific heats, $\gamma_{H}$, has been fixed such that the value in the lower layer corresponds to $\gamma_{L}=1.4 ; \gamma(z)$ decreases with increasing $z^{*}$ according to Eq. (87) until $\gamma_{H}=1.1$ at the top of the domain.

The equations are solved in a rectangular domain $2 L_{T}^{*}$ $\times 2 L_{T}^{*} \times 11 L_{T}^{*}$, where $L_{T}^{*}=L_{T} / L_{H}=\ln \left(W_{H} / W_{L}\right)$. This size is chosen such that the upper half of the domain spans six times the distance $L_{T}$ and the lower half of the domain five times. This length scale $L_{T}$, defined by

$$
L_{T}=L_{H} \ln \frac{L_{L}}{L_{H}},
$$

is the important scale of this configuration, as shown by Eqs. (43) and (44), rather than $L_{H}$, because it retains information about the density jump at the interface.

The mesh size is $128 \times 128 \times 704$. The initial thickness is set to $h_{Y, 0}^{*}=0.171$, so that there are ten points to resolve the initial gradient. The initial perturbation field $\xi^{*}(x, y)$ is set following Cook et al. ${ }^{13}$ by considering a Gaussian distribution of energy content in its two-dimensional spectrum, with the peak located at a wavelength $\lambda_{0}^{*}=L_{T}^{*} / 6$. The standard deviation in frequency space is $f_{0}^{*} / 6$, where $f_{0}^{*}=1 / \lambda_{0}^{*}$, so that

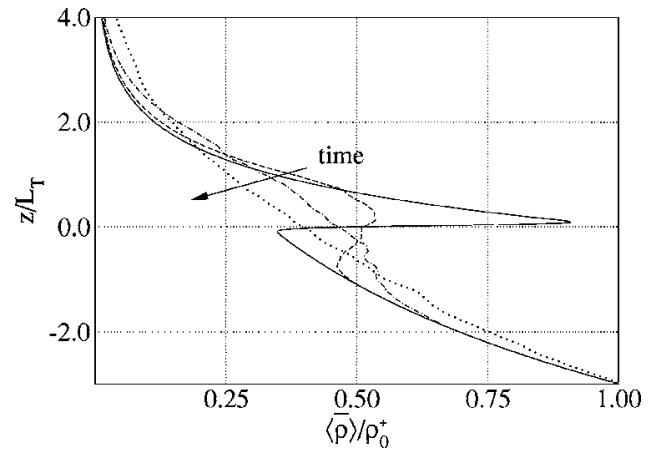

FIG. 4. Normalized mean density profiles at different times: $(-) t \sqrt{A g / L_{T}}$ $=0 ;(--)) t \sqrt{A g / L_{T}}=3.5\left(h_{Y} / L_{T}=2\right) ;(-\cdot) t \sqrt{A g / L_{T}}=6.9\left(h_{Y} / L_{T}=4\right) ;(\cdots \cdots \cdots \cdot)$ $t \sqrt{A g / L_{T}}=12.0\left(h_{Y} / L_{T}=8\right)$.

there is practically no energy in the frequency range between 0 and $f_{0}^{*} / 2$. The root-mean-squared value of the perturbation is $10 \%$ of $\lambda_{0}^{*}$, which implies $\xi_{\text {rms }}^{*} / h_{Y, 0}^{*}=0.1$.

\section{B. Density field}

Figure 4 presents the vertical profiles of the normalized mean density. In addition to the initial distribution, the density variation at times $t \sqrt{A g / L_{T}}$ equal to $3.5,6.9$, and 12.0 is plotted. The mixing zone thickness $h_{Y}$ at each of these times is also indicated. This figure shows that the density jump at $z=0$ is rapidly reduced due to the mixing process in a time that scales with $\sqrt{L_{T} /(\mathrm{Ag})}$, the instantaneous Atwood number based on the mean density decreasing with time. By the time that the mixing layer grows to $h_{Y} / L_{T}=4$, the regions with static instability of the mean density profile have practically disappeared, clearly showing that the mixing by RayleighTaylor turbulence is restricted to a central zone that scales with the length $L_{T}$, as assumed in the theoretical analysis of Sec. II A.

Figure 5 shows the temporal evolution of the normalized intensity of the density fluctuations at the center plane, defined as

$$
\frac{A_{e}}{A}=\frac{\rho_{\mathrm{rms}}}{\langle\bar{\rho}\rangle A} .
$$

The maximum value at late times is only slightly larger than the center plane value presented in this figure and occurs in

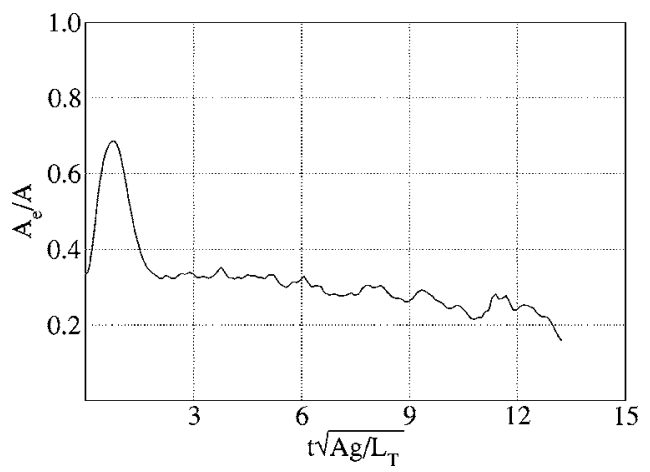

FIG. 5. Temporal evolution of the normalized intensity of the density fluctuations or effective Atwood number, Eq. (92), at the center plane. 


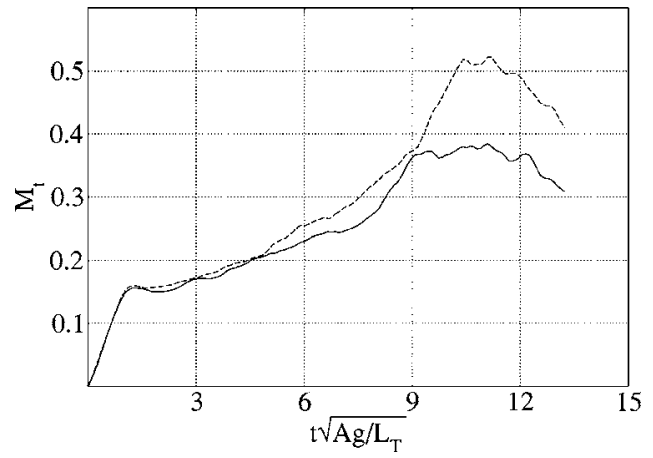

FIG. 6. Temporal evolution of the turbulent Mach number, $M_{t}=q /\langle c\rangle:-$ at the center plane, --- maximum value.

the lower layer of fluid, where eventually the density is higher than in the whole upper layer. This quantity is the effective Atwood number of the flow, $A_{e}$, as proposed by Cook et al. ${ }^{15}$ The initial peak for $t \simeq \sqrt{L_{T} /(A g)}$ represents the increase in segregation during the initial development of the flow, before turbulence dominates the problem. After transition, the effective Atwood number decreases continuously due to the stable stratification of the upper and the lower layers, which confines the region of fluid involved in the problem to a finite size, and turbulent mixing, which eventually mixes both species at the molecular level.

\section{Intrinsic compressibility}

Figure 6 shows the temporal evolution of the turbulent Mach number. Along with the value at the center plane, the maximum value, which occurs somewhat off-center toward the upper layer of fluid where the speed of sound is lower, is also plotted.

The fact that the mean density evolves in a scale of order $L_{T}$ as observed in Fig. 4 confirms the overall available potential energy calculated in the theoretical analysis. The speed of sound was estimated by the mean final value and it is observed that $M_{t}$ at the center plane in the LES is less than the upper bound found in Sec. II A, $\approx 0.6$, confirming the analysis. There is a displacement of the maximum value of $M_{t}$ toward the initially heavy fluid side (upper layer), but the difference observed in Fig. 6 is small enough for the bounds obtained in Sec. II A to hold in general.

It has to be underlined as well that the initial perturbation of the interface is chosen small compared to $L_{T}$, so that fully developed turbulence could be established as much as possible before $h_{Y}(t)$ reaches values of the order of $L_{T}$. However, if a large perturbation is set initially so that a largescale velocity field develops in the beginning with little (small-scale) mixing, there could be stronger compressibility effects as the upper layer, with low speed of sound, is strongly perturbed in the areas of pure heavy fluid. This is not the case considered in this study, not because it is less important, but because that situation would be strongly problem dependent.

In order to further analyze the compressibility of the flow, apart from looking at the turbulent Mach number, it is also customary to split the density fluctuation shown in Fig. 5 into an acoustic part and an entropic part, one possible definition being ${ }^{23}$

$$
\begin{aligned}
& \rho_{\mathrm{ac}}^{\prime}=p^{\prime} /\langle c\rangle^{2}, \\
& \rho_{\mathrm{en}}^{\prime}=\rho^{\prime}-\rho_{\mathrm{ac}}^{\prime} .
\end{aligned}
$$

Here, since the flow is practically isothermal, the entropic part originates from the composition fluctuations. Results show that the major part of the fluctuation $\rho_{\mathrm{rms}}$ shown in Fig. 5 corresponds to the entropic mode, the acoustic contribution being only $6 \%$ of the entropic one at the center plane at $t \sqrt{\mathrm{Ag} / L_{T}}=3.5$, which is characteristic of a situation with low intrinsic compressibility. At later times, this ratio slightly increases, being $10 \%$ at $t \sqrt{A g / L_{T}}=6.9$, which is still a small value. Consistently, the pressure-dilatation term in the transport equation for the turbulent kinetic energy (to be discussed more thoroughly for the next configuration) is less than $10 \%$ of the buoyancy-production term.

The results of the LES thus confirm the theoretical predictions of the bounds of the turbulent Mach number. The value of $M_{t}$ in the turbulent stage of the Rayleigh-Taylor problem does not have significant intrinsic compressibility effects.

\section{SIMULATION OF THE CONFIGURATION WITH EACH LAYER AT CONSTANT DENSITY}

\section{A. Problem definition}

The configuration shown in Fig. 3 and analyzed in Sec. II $\mathrm{C}$ is now considered. The density has a smooth transition from the lower to the higher value over a thickness $h_{\rho, 0}$ and the temperature is constant everywhere. Initially, the system has no fluctuations. At time $t=0$ the interface between the two fluids is perturbed and the temporal development of the resultant Rayleigh-Taylor instability into turbulence is simulated.

The problem is nondimensionalized with $p_{0}, g$ and the thermodynamic state of the pure heavy fluid, $\rho_{H}$ and $T_{H}$. A length scale is given by $L_{H}$, Eq. (67), and velocities are nondimensionalized with $\sqrt{g L_{H}}$ and molecular weights by $W_{H}^{+}$ $=R^{0} T_{H} \rho_{H} / p_{0}$. The nondimensional governing equations are the same as in the preceding section, the only parameter entering in those transport equations being now the reference ratio of specific heats $\gamma_{H}^{+}=1 /\left[1-R^{0} /\left(C_{p} W_{H}^{+}\right)\right]$.

The setup is a vertically varying mixture of two species such that the profile of the density is given by

$$
\rho^{*}\left(z^{*}\right)=\frac{1+\rho_{L}^{*}}{2}\left[1+A \operatorname{erf}\left(\frac{z^{*}+\xi^{*}(x, y)}{2 h_{\rho, 0}^{*} / 6.58}\right)\right],
$$

where $h_{\rho, 0}^{*}$ is the initial thickness of the mixing layer and the initial perturbation is described by $\xi^{*}(x, y)$. The thickness $h_{\rho}$ is defined by the length over which the mean density profile varies from $1 \%$ of the density jump above $\rho_{L}^{*}$ to $1 \%$ of the density jump below $\rho_{H}^{*}=1$. Again, the asterisk denotes nondimensional values.

The nondimensional pressure is obtained from Eq. (66), 


$$
p^{*}\left(z^{*}\right)=1-\int_{0}^{z^{*}} \rho^{*}(\zeta) d \zeta
$$

The nondimensional parameters defining the isothermal problem are $h_{\rho, 0}^{*}=h_{\rho, 0} / L_{H}, \rho_{L}^{*}=\rho_{L} / \rho_{H}$ [or, equivalently, the Atwood number, Eq. (3)], the molecular weights of the pure species $W_{L}^{*}=W_{L} / W_{H}^{+}$and $W_{H}^{*}=W_{H} / W_{H}^{+}$(which determine the profiles of species mass fractions), and $\gamma_{H}^{+}$. The effect of the density ratio on compressibility has already been taken into account in the analysis presented in Sec. II. The last three nondimensional parameters are coupled with the velocity field only through $\gamma$, as explained in Sec. III, and their influence in the results is then expected to be small. With respect to the initial thickness $h_{\rho, 0}^{*}$, it is desirable to set it as small as possible so that a self-similar turbulent stage is achieved, but, on the other hand, it determines the resolution required in the LES. Its possible effect on compressibility is studied in the following section.

The density ratio considered in this simulation is $\rho_{H} / \rho_{L}=3$ (Atwood number $A=0.5$ ). The reference ratio of specific heats has been fixed such that the value in the pure light fluid is $\gamma_{L}=1.4 ; \gamma(z)$ then varies from $\gamma=1.33$ at the bottom to $\approx 1$ at the top of the domain.

The equations are solved in a rectangular domain $L_{H}$ $\times L_{H} \times 2 L_{H}$. Thus, the vertical size of the upper layer, $L_{H}$, is the maximum possible according to the definition of the scale-height, Eq. (67), and is chosen to obtain the maximum achievable velocity fluctuation in this configuration. The pressure is not exactly zero at the top boundary because of the initial thickness $h_{\rho, 0}^{*}$ at the center plane. The mesh size is $256 \times 256 \times 512$, double the resolution of the case considered in Sec. IV. The motivation for this larger simulation is the attempt to reach self-similar behavior in some of the statistics, as will be discussed below. The initial thickness is set to $h_{\rho, 0}^{*}=0.039$, so that there are ten points to resolve the initial gradient. The initial perturbation field $\xi^{*}(x, y)$ is set as explained in Sec. IV, scaled accordingly to the smaller characteristic scale of the initial perturbation $h_{\rho, 0}^{*}$ considered in this case. Thus, the peak of the spectral distribution of the perturbation is $\lambda_{0}^{*}=1 / 24$.

\section{B. Intrinsic compressibility}

The results describing the intrinsic compressibility of the flow are now presented. It is interesting to note that the coefficient $\beta$ of Eq. (10) is 0.36 in the simulation, based on the Reynolds stresses obtained in the LES, to be discussed later, Fig. 14. This result is close to the value 0.3 reported by Cook et al. ${ }^{15}$ for the incompressible case. Furthermore, negligible explicit compressibility effects in the following sections will be seen, recovering scaling laws and quantitative results reported in incompressible situations.

The first quantity to be examined is the turbulent Mach number, defined by Eq. (4). Its temporal evolution at the center plane, where it approximately peaks, is represented by the solid curve in Fig. 7, observing a maximum value about 0.15 . Note that the computational box extends to a height $L_{H}$ above the center plane, the maximum available to ensure positive pressure. Consequently, the maximum value of tur-

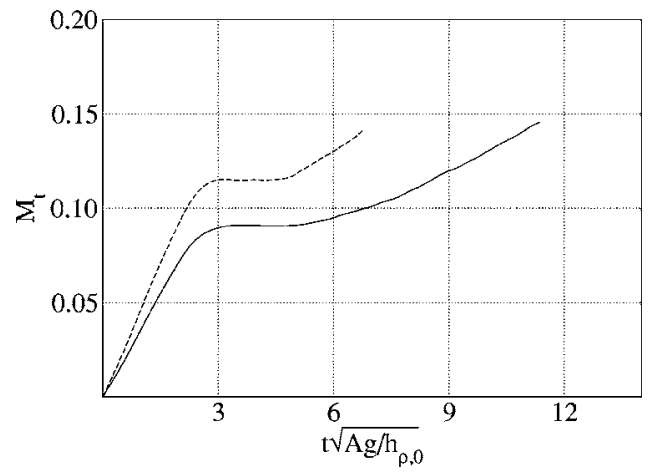

FIG. 7. Temporal evolution at the center plane of the turbulent Mach number, $M_{t}=q /\langle c\rangle$ : (一) $h_{\rho, 0} / L_{H}=0.039$ (mesh $\left.256 \times 256 \times 512\right) ;(--) h_{\rho, 0} / L_{H}$ $=0.078($ mesh $128 \times 128 \times 256)$.

bulent Mach number achieved in the simulation is a good estimate of the maximum $M_{t}$ achievable in this configuration. This result of $M_{t, \max }=0.15$ is consistent with the bounds obtained in Sec. II. The smallness of $M_{t}$ implies that compressibility effects are expected to be too weak to alter the general characteristics of the flow.

In addition to the solid curve, there is a dashed curve in Fig. 7 corresponding to another LES that only differs in the size of the initial mixing depth, $h_{\rho, 0}^{*}=0.078$ (instead of $h_{\rho, 0}^{*}$ $=0.039$ ) and the mesh size, $128 \times 128 \times 256$. As mentioned in the beginning of this section, the size of the initial perturbation is the second relevant nondimensional parameter defining the problem (the first one, $\rho_{L} / \rho_{H}$, has been considered in Sec. II) and its influence on the achievable turbulent Mach number is not easy to analyze theoretically. Both simulations stop when the mixing thickness becomes $h_{\rho}^{*} \simeq 0.7$ because the domain size affects the results beyond that point. The consequence of this difference in the initial conditions is that $M_{t}$ increases less during the initial developing stage up to three nondimensional time units, leaving more time for the turbulent evolution, as the size of the initial perturbation $h_{\rho, 0}^{*}$ is decreased. However, the final value maximum $M_{t} \simeq 0.15$ is the same in both cases.

As it was done in the two-layer configuration, Sec. IV, the density fluctuation was split into an acoustic part and an entropic part, as defined by Eq. (93). Results show that almost all the fluctuation $\rho_{\text {rms }}$ correspond again to the entropic mode, the acoustic part being only $2.5 \%$ of the entropic part, confirming a quasi-incompressible situation.

\section{Comparison with previous work}

As mentioned in Sec. II C, the choice of a configuration formed by a constant density layer over another constant (but lighter) density layer is partly motivated by the possibility of comparison with previous work and, thus, validation of the LES. We now briefly discuss subgrid model coefficients, the evolution of the thickness $h_{\rho}$, and molecular mixing.

The subgrid model coefficient obtained in the later stages of the simulation is $C_{u} \simeq 0.012$, which agrees well with the range $0.002-0.02$ found in the literature for other flows. ${ }^{35,38,39,48,49}$ The value of the subgrid Prandtl number, $\operatorname{Pr}_{\mathrm{sg}}=C_{u} / C_{p} \simeq 0.35$, is somewhat lower than the range $\operatorname{Pr}_{\mathrm{sg}}$ 


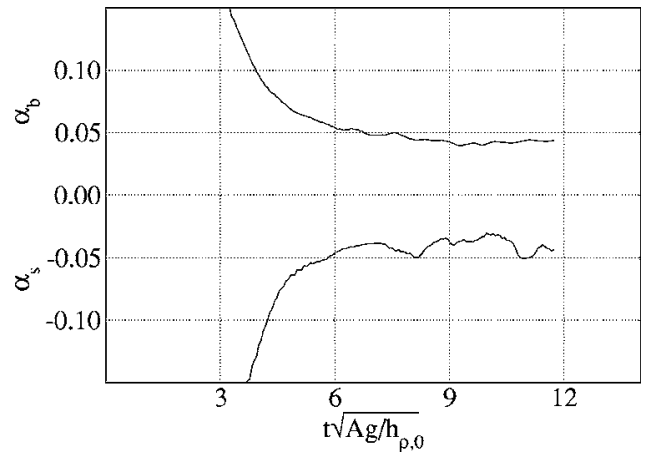

FIG. 8. Growth coefficients: $\alpha_{b}$ for bubbles (positive values) and $\alpha_{s}$ for spikes (negative values).

$\simeq 0.4-0.85$ reported for a passive scalar. The subgrid Schmidt number, $\mathrm{Sc}_{\mathrm{sg}}=C_{u} / C_{Y}$, follows closely the evolution of $\operatorname{Pr}_{\mathrm{sg}}$. This relatively broad range of values is reasonable because, in principle, the coefficients could depend on the filter kernel, the closure model (dynamic or dynamic mixed), and the type of flow, and there could be an additional dependence on the Reynolds number and the ratio $\Delta_{f} / L$ of the data reported by different authors.

The mixing depth based on $h_{\rho}$ was studied by the growth coefficients of the bubble penetration, $\alpha_{b}$, and the spikes penetration, $\alpha_{s}$, as defined in the Introduction. Figure 8 plots the time derivative of the bubble penetration, $h_{\rho, b}(t)$, and the spike penetration, $h_{\rho, s}(t)$, with respect to $\left(A g t^{2}\right)$, following Cook et al. ${ }^{13} \mathrm{~A}$ tendency toward a constant value, confirming a quadratic time dependence of the mixing depth, is observed. The intrinsic compressibility does not affect this quadratic growth law because the value of $M_{t}$ remains rather small, as seen previously. The final value of $\alpha_{b}$ and $\alpha_{s}$, about 0.04 , is consistent with the data found in the literature.

Lastly, mixing is investigated. Mixing has been characterized in the past in several manners. Two depth-integrated parameters are considered, both based on the ratio

$$
\frac{\int\left\langle X_{p}(X)\right\rangle d z}{\int X_{p}(\langle X\rangle) d z} .
$$

The particular choice of the function $X_{p}$ leads to one parameter or another. In this expression, $X=\left(\rho-\rho_{L}\right) /\left(\rho_{H}-\rho_{L}\right)$, which in the incompressible limit represents the mole fraction of the heavy fluid. We compute first the mixing parameter $\Theta$ as proposed by Youngs, ${ }^{12}$

$$
X_{p}=X(1-X),
$$

and second, the mixing parameter $\Xi$ as proposed by Cook et al., ${ }^{13}$

$$
X_{p}= \begin{cases}2 X, & X \leq 1 / 2 \\ 2(1-X), & X>1 / 2 .\end{cases}
$$

The temporal evolution of both parameters is presented in Fig. 9. It is observed that they evolve very close to each other, either one giving the same information and following the general temporal evolution described by the aforemen-

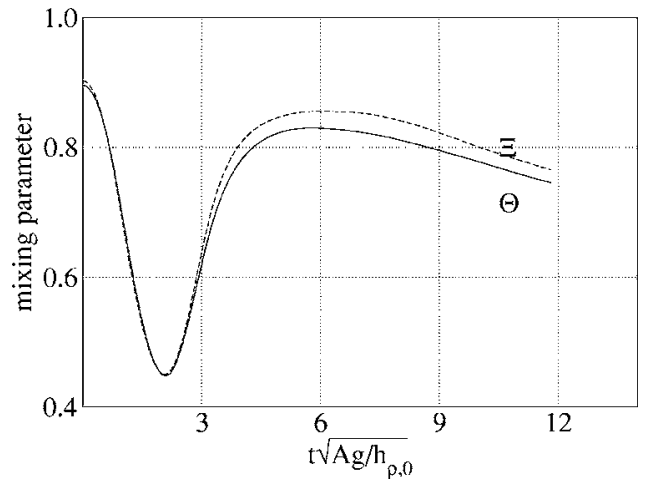

FIG. 9. Temporal evolution of the global mixing parameters: $\Theta$-Youngs' definition, $\Xi$-Cook's definition.

tioned authors. The final values in the current LES, although still slightly evolving, are about 0.75 , which agree reasonably well with the result 0.8 reported in both of the previous references and elsewhere. ${ }^{11,15,22}$ Again, there is no obvious influence of intrinsic compressibility on the mixing parameters.

\section{Density field}

The mixing layer is usually measured by the distance $h_{\rho}$ over which the density profile varies from $1 \%$ over $\rho_{L}$ to $1 \%$ below $\rho_{H}$. However, this measure of the mixing depth is not very robust, especially with relatively small domains, since isolated structures (bubbles/spikes) at the fronts can change it and the temporal evolution is not very regular. ${ }^{14}$ For this reason, we choose the mixing depth defined as

$$
\delta_{\rho}=\frac{\Delta \rho}{(\partial\langle\bar{\rho}\rangle / \partial z)_{\max }},
$$

which is equivalent to the vorticity thickness used in the mixing layer, a problem where the velocity jump drives the flow instead of a jump in the density. The initial relation between $\delta_{\rho}$ and the alternate mixing height $h_{\rho, 0}$, defined by Eq. (94), is $\delta_{\rho, 0}=0.54 h_{\rho, 0}\left(\delta_{\rho, 0}^{*}=0.021\right)$ in this particular case.

In order to determine $(\partial\langle\bar{\rho}\rangle / \partial z)_{\max }$ at a given time, a fifth-order polynomial is fitted to the data of the mean density profile between $\rho_{L}+0.01 \Delta \rho$ and $\rho_{H}$, and the derivative is obtained using second-order centered finite differences. Figure 10 shows the evolution of the mixing width $\delta_{\rho}(t)$ and its time derivative $\dot{\delta}_{\rho}(t)$. Time has been nondimensionalized by

$$
\tau=\sqrt{\delta_{\rho, 0} /(A g)} .
$$

Note that the initial irregularities in $\dot{\delta}_{\rho}(t)$ do not persist at late time, the period of most interest.

Theory for the low Mach number situation predicts that $\delta_{\rho} \propto t^{2}$ once the self-similar stage is achieved. This behavior is tested more easily with the derivative $\dot{\delta}_{\rho}(t)$, which should vary linearly with time. The figure shows that there is an initial period, $t<2 \tau$, where the increase of $\delta_{\rho}$ is roughly quadratic. After this initial development, there is an interval between a nondimensional time of 2.5 and 5 where the average flow decelerates. Once this initial transient is over- 


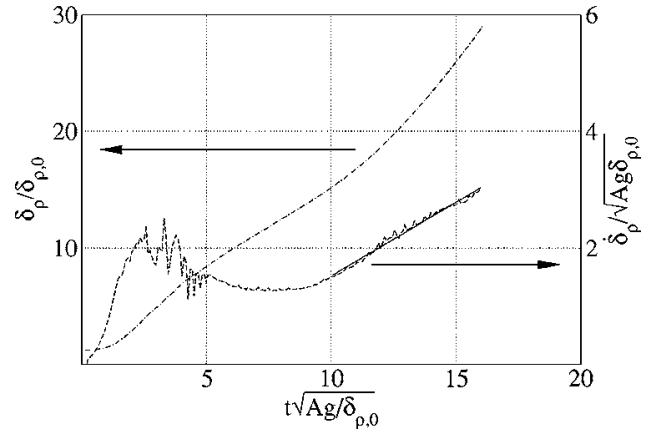

FIG. 10. Mixing width $\delta_{\rho}$ (dash-dotted line) and its time derivative $\dot{\delta}_{\rho}$ (dashed line) as a function of time. The linear fit to $\dot{\delta}_{\rho}$, Eq. (101), is also shown as a solid line.

come, $\delta_{\rho}$ starts to accelerate again, seemingly toward the quadratic scaling. The linear fit to the data beyond $t=10 \tau$ provides

$$
\dot{\delta}_{\rho} / \sqrt{A g \delta_{\rho, 0}}=0.25(t / \tau-3.96) .
$$

Again, intrinsic compressibility does not influence the usual scaling law of the growth of $\delta_{\rho}$ that neglects any Mach number influence. At the nondimensional time of 16, the thickness of the mixing layer is $\delta_{\rho}^{*} \simeq 0.61\left(h_{\rho}^{*} \simeq 0.72\right)$. The simulation is then stopped because the vertical extent is comparable to the homogeneous dimensions (recall that the domain size is $L_{H} \times L_{H} \times 2 L_{H}$ ) and the number of structures in the domain does not allow a reasonable average.

Figure 11 plots the density profile across the flow for two different times, $t=10 \tau$ and $t=15 \tau$. The vertical coordinate is scaled with the mixing depth $\delta_{\rho}$, and the collapse of the two curves suggests an approach to similarity. There is a small deviation, however, at the two fronts; it is not clear if the deviation is due to the LES model or truly due to the lack of self-similarity. This deviation will be shown to be stronger in the Reynolds stresses profiles.

The last density-related quantity to be analyzed is the density fluctuation variance, cast in terms of an effective Atwood number, Eq. (92). Its temporal evolution at the center plane is shown in Fig. 12. The peak occurring at $t$ $\simeq 2.5 \tau$ represents an increase in segregation taking place in the nonlinear stage (minimum of the mixing parameters in Fig. 9). After that, the sustained increase of density fluctua-

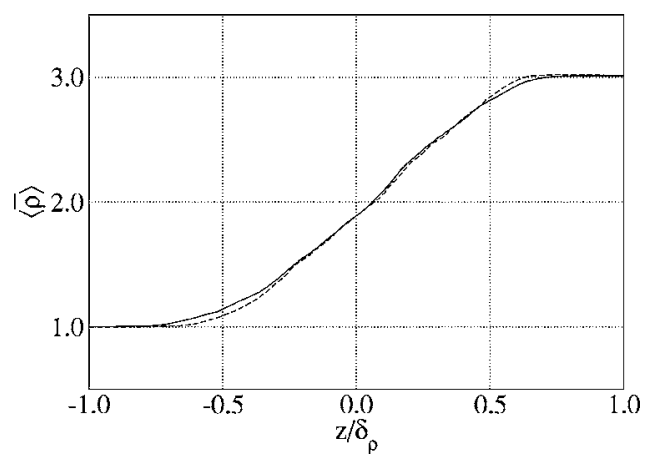

FIG. 11. Mean density profile using self-similar variables: (-) $t$ $=10 \sqrt{\delta_{\rho, 0} /(A g)} ;(--) t=15 \sqrt{\delta_{\rho, 0} /(A g)}$.

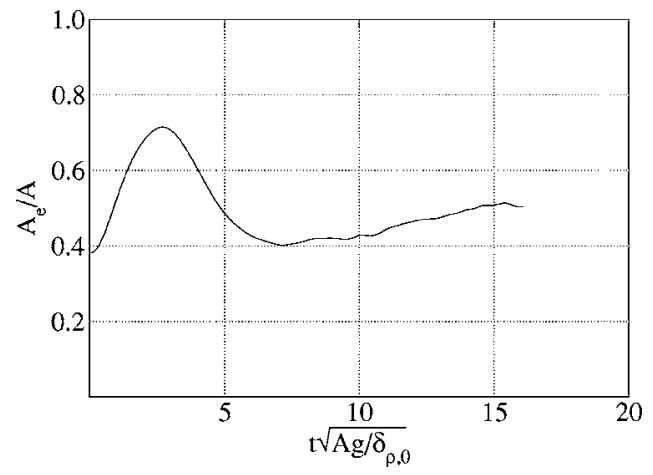

FIG. 12. Temporal evolution of the normalized intensity of the density fluctuations or effective Atwood number, Eq. (92), at the center plane.

tions beyond transition is due to the unstable stratification (negative buoyancy-frequency) of the upper and the lower layers, consistently the opposite tendency from the earlier case, Fig. 5, where the stratification was stable. This result is also consistent with the sustained decrease in the mixing parameters shown in Fig. 9.

The information given by Fig. 12 is relevant for the topic investigated in this paper. First, miscible fluids are characterized by an effective Atwood number significantly smaller than immiscible fluids. For immiscible fluids, i.e., completely segregated states, the effective Atwood number is approximately ${ }^{15}$ equal to $A$, or possibly larger because of the unstable stratification in each layer. Miscible fluids are characterized by a smaller value in the turbulent stage, in particular, we obtain $A_{e}=0.5 \mathrm{~A}$, which is in agreement with the incompressible case considered by Cook et al. ${ }^{15}$ Figure 13, the visual counterpart of the information conveyed by the curve in Fig. 12, shows that the late-time mixing (bottom panel) is turbulent; there is a central region of intermediate fluctuating values of density, and the probability of bubbles or spikes enclosing pure fluid and cleanly penetrating in the opposite layer is small. Turbulent mixing also justifies the choice of the characteristic speed of sound in the theoretical analysis to be a mean value of the speed of sound in the turbulent core.

Second, the effect of compressibility can be directly observed comparing Fig. 12 with Fig. 19 in the work of Cook et al. ${ }^{15}$ These authors considered the incompressible case and they observed a constant behavior of $A_{e}$ for late times due to stable stratification. Compressibility imposes a negative buoyancy-frequency and thus unstable stratification, which

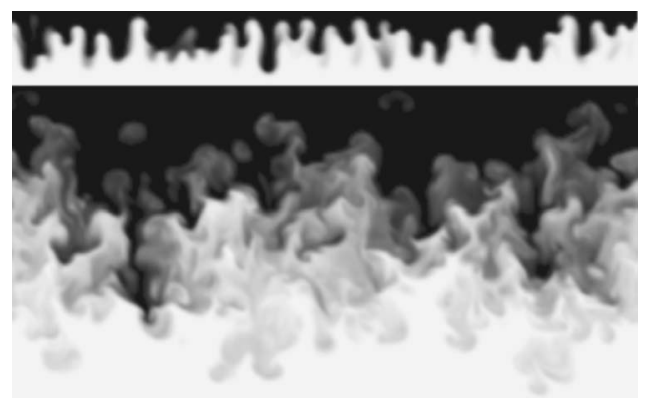

FIG. 13. Density fields. Top $-t=2.5 \tau$ (nonlinear stage); bottom $-t=10 \tau$ (turbulent stage). Gravity is acting downward. 


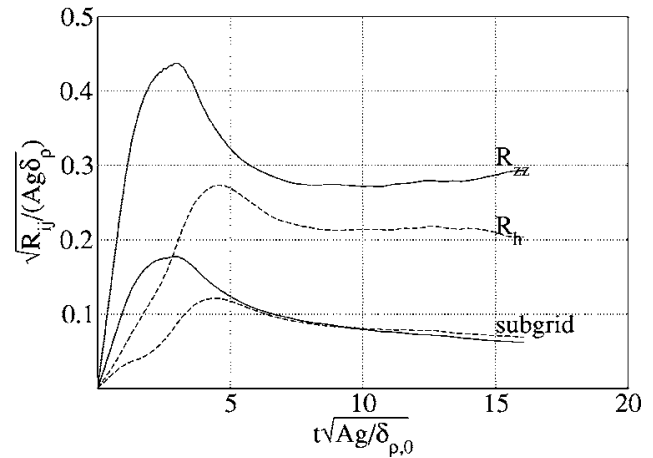

FIG. 14. Temporal evolution at the center plane of the total Reynolds stresses: (-) vertical, $R_{z z}$; (---) horizontal, $R_{h}=R_{x x}+R_{y y}$. The bottom curves denote, with the same line pattern, the corresponding subgrid parts.

results in the increase of the density fluctuations. The increase in the effective Atwood number due to this buoyancycompressibility coupling is of the order of $20 \%$.

\section{E. Reynolds stresses}

The Reynolds stress tensor is split into resolved, and subgrid parts,

$$
R_{i j}=R_{i j}^{r}+R_{i j}^{\mathrm{sg}},
$$

where

$$
\begin{aligned}
& R_{i j}^{r}=\left\langle\bar{\rho} \bar{u}_{i}^{\prime} \bar{u}_{j}^{\prime}\right\rangle /\langle\bar{\rho}\rangle, \\
& R_{i j}^{\mathrm{sg}}=\left\langle q_{u, i j}\right\rangle /\langle\bar{\rho}\rangle,
\end{aligned}
$$

the subgrid contribution being estimated from the same dynamic mixed model used in the LES. The previous relation between the total, resolved, and subgrid stresses holds if the filter size $\Delta_{f}$ is small enough to allow the approximation $\overline{\langle\phi\rangle} \simeq\langle\phi\rangle$.

Figure 14 shows the temporal evolution at the center plane of the total Reynolds stresses, as defined above, nondimensionalized with the velocity scale $\sqrt{A g \delta_{\rho}}$. The horizontal Reynolds stress is defined by $R_{h}=R_{x x}+R_{y y}$. The temporal evolution shows the transient seen in the mixing depth, with a strong peak in the vertical Reynolds stress $R_{z z}$. It is also observed that the horizontal stress has certain delay with respect to the vertical one, the peak occurring at later times, because the input of energy is through $R_{z z}$ followed by a transfer to $R_{h}$. The tendency toward a self-similar stage is also approximately seen beyond the nondimensional time 8.0 , where the plateau in the curves confirms the expected scaling with the velocity $\sqrt{\operatorname{Ag} \delta_{\rho}}$. However, there is still a slight increase in anisotropy.

Figure 15 plots the profiles at the times $t=10 \tau$ and $t$ $=15 \tau$ using the similarity variable $z / \delta_{\rho}$. This figure shows a clear asymmetry in the flow, the peak of the Reynolds stresses being slightly displaced toward the light fluid. It is also observed that the profiles do not collapse very well, especially in the spikes front (negative $z$ ), similar to the density profiles.

The level of anisotropy of the Reynolds stresses measured by the ratio between the vertical and the horizontal

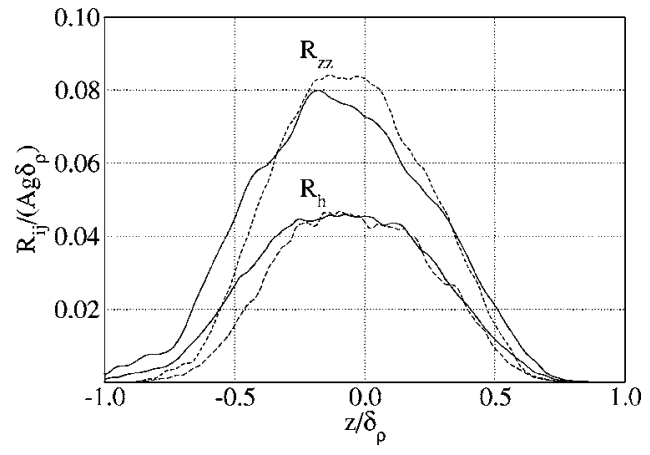

FIG. 15. Total Reynolds stresses profiles using self-similar variables: (一) $t=10 \sqrt{\delta_{\rho, 0} /(A g)} ;(--) t=15 \sqrt{\delta_{\rho, 0} /(A g)}$.

fluctuations is $R_{z z} / R_{h} \simeq 1.8$, as can be observed in Fig. 15 . This result is in agreement with the value 1.7 reported by Cook et al. ${ }^{15}$ and Dimonte et al., ${ }^{22}$ and 1.5 reported by Linden $e t$ al. ${ }^{16}$ This anisotropy is larger than that found in free jets or mixing layers, ${ }^{50}$ where the ratio of the streamwise intensity to the cross-sectional fluctuation is closer to unity, suggesting that a directional volumetric force induces more anisotropy than a directional shear as driving mechanism.

The subgrid Reynolds stresses, as obtained from the dynamic mixed model, Eq. (85), are shown in Fig. 14. The contribution of the Smagorinsky part to the Reynolds stresses is negligible, $\approx 1 \%$ of the scale similarity part. Their temporal evolution is similar to that of the resolved counterparts. The subgrid kinetic energy at the end of the simulation is about $7 \%$ of the total amount, decreasing with time.

It is interesting to see how the latter ratio $K^{\mathrm{sg}} / K$ varies with $L_{\varepsilon} / \Delta_{f}$, where the integral dissipation length is defined by $L_{\varepsilon}=K^{3 / 2} / \varepsilon^{\mathrm{sg}}, \varepsilon^{\mathrm{sg}}$ being the mean subgrid-scale dissipation. This dissipation scale achieves an approximate constant value of $L_{\varepsilon}=0.3 \delta_{\rho}$ beyond $t=10 \tau$. Figure 16 shows the expected decay, as the energy is continuously displaced toward the larger resolved sales. Bagget et al. ${ }^{51}$ reports smaller subgrid contribution for smaller ratios of $L_{\varepsilon} / \Delta_{f} \simeq 10$ in a channel flow, but this is consistent with the fact that their spectral cut-off filter yields more energy in the resolved part of the spectrum. Besides, some dependence on the specifics of the flow might be expected.

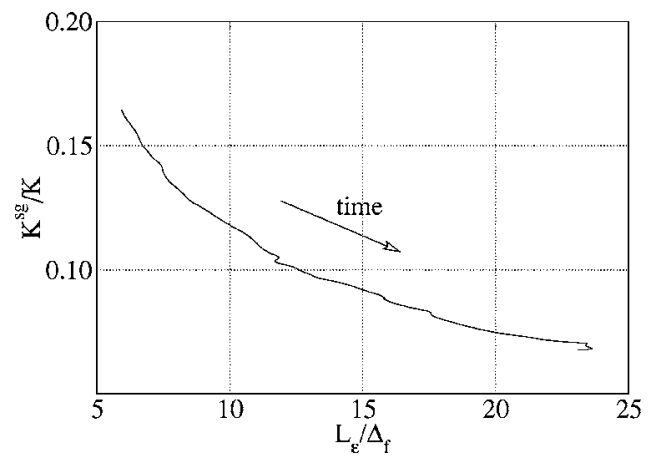

FIG. 16. Variation of the percentage of subgrid energy as the filter size $\Delta_{f}$ decreases in comparison with the dissipation length scale, $L_{\varepsilon}=K^{3 / 2} / \varepsilon^{\mathrm{sg}}$. 


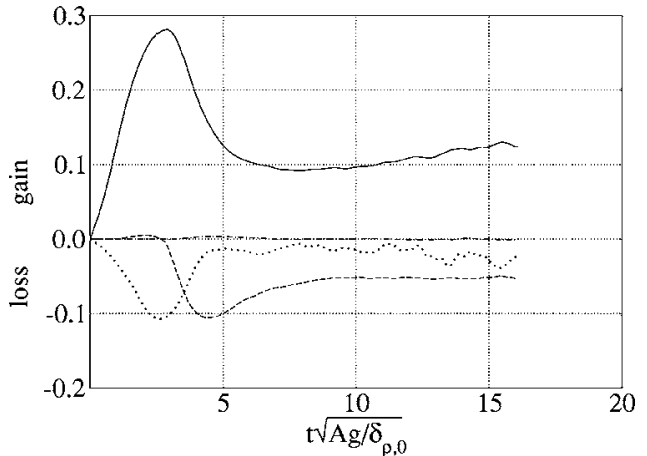

FIG. 17. Temporal evolution at the center plane of the budget of the resolved turbulent kinetic energy equation per unit mass, nondimensionalized by $\sqrt{(A g)^{3} \delta_{\rho}}$ : (一) buoyancy production, $B^{r} /\langle\bar{\rho}\rangle$; (---) subgrid dissipation, $-\varepsilon^{\mathrm{sg}} ;(\cdots \cdots \cdots \cdot)$ transport, $-T_{j, j}^{r} /\langle\bar{\rho}\rangle ;(-\cdot)$ pressure-dilatation, $\Pi^{r} /\langle\bar{\rho}\rangle$.

\section{F. Turbulent kinetic energy budget}

The transport equation for the resolved turbulent kinetic energy per unit mass, $K^{r}$, reads

$$
\begin{aligned}
\langle\bar{\rho}\rangle \frac{\partial K^{r}}{\partial t}+\langle\bar{\rho}\rangle\langle\bar{w}\rangle \frac{\partial K^{r}}{\partial z}+\frac{\partial T_{z}^{r}}{\partial z}= & \Pi^{r}+\langle\bar{\rho}\rangle\left(P^{r}-\varepsilon^{\mathrm{sg}}\right)+B^{r} \\
& +\Sigma^{r},
\end{aligned}
$$

where

$$
\begin{aligned}
& T_{z}^{r}=\left\langle\bar{\rho} \bar{w}^{\prime} \bar{u}_{i}^{\prime 2}\right\rangle / 2+\left\langle\bar{p}^{\prime} \bar{w}^{\prime}\right\rangle+\left\langle\bar{w}^{\prime} q_{u, z z}^{\prime}\right\rangle, \\
& \Pi^{r}=\left\langle\bar{p}^{\prime} \bar{u}_{i, i}^{\prime}\right\rangle, \\
& P^{r}=-R_{z z}\langle\bar{w}\rangle_{, z}, \\
& \varepsilon^{\mathrm{sg}}=-\left\langle q_{u, i j}^{\prime} \bar{u}_{i, j}^{\prime}\right\rangle /\langle\bar{\rho}\rangle, \\
& B^{r}=\left\langle\bar{\rho}^{\prime}(\bar{w})_{R}^{\prime}\right\rangle\langle\bar{p}\rangle_{, z}\langle\bar{\rho}\rangle, \\
& \Sigma^{r}=\left\langle\bar{\rho}^{\prime}(\bar{w})_{R}^{\prime}\right\rangle\left\langle q_{u, z z}\right\rangle_{, z} /\langle\bar{\rho}\rangle .
\end{aligned}
$$

In these expressions, $(\bar{w})_{R}^{\prime}$ denotes the Reynolds fluctuation of the vertical velocity and $B$ is the buoyancy term. This equation is obtained from the first two transport equations in the system, Eq. (81). In comparison with the transport equation for the total turbulent kinetic energy, it does not contain any of the terms due to molecular diffusion, and it includes instead the subgrid-scale part in the transport $T_{z}^{r}$ and the mean flux $\Sigma^{r}$, in addition to the mean subgrid-scale dissipation $\varepsilon^{\mathrm{sg}}$.

Figure 17 shows the temporal evolution at the center plane of the most significant terms in Eq. (104). The subgrid part of the transport term is approximately constant beyond $t=10 \tau$ while the resolved part increases so that the ratio follows a trend similar to $K^{\mathrm{sg}} / K$, Fig. 16 , being about $7 \%$ at the end of the simulation. It is clear that the main input of energy in this flow is the buoyancy term $B^{r}$. Note that $\langle\bar{p}\rangle_{, z}$ $\simeq-\langle\bar{\rho}\rangle g$ is negative, and so is the mass flux $\left\langle\bar{\rho}^{\prime}(\bar{w})_{R}^{\prime}\right\rangle$ because heavy fluid pockets in lighter ambient fluid drop while light pockets in heavy fluid rise. Therefore, the buoyancy term is positive and represents turbulent potential energy being

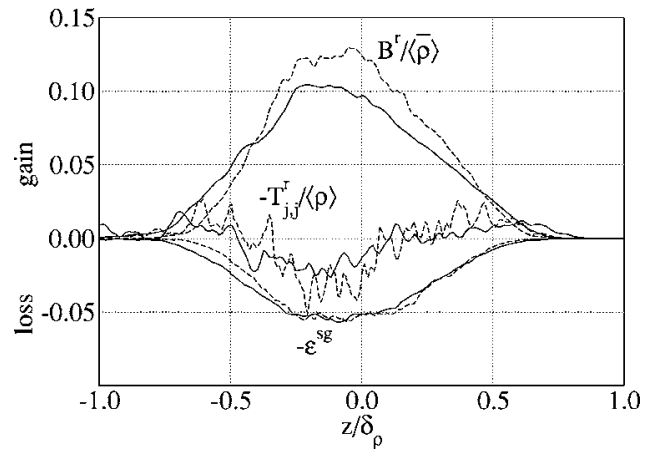

FIG. 18. Budget of the resolved turbulent kinetic energy equation, nondimensionalized by $\sqrt{\left(A g^{3}\right) \delta_{\rho}}:(-) t=10 \sqrt{\delta_{\rho, 0} /(A g)} ;(--) t=15 \sqrt{\delta_{\rho, 0} /(A g)}$.

transferred to turbulent kinetic energy (in other cases, e.g., stable stratification, the transfer could be in the opposite direction). Production and convection play a small role because the mean velocity and its gradient are small. The pressure-dilatation term is negligible, which further indicates the quasi-incompressible character of the flow.

The energy is distributed in space by the transport term $T_{z, z}^{r}$, moving energy from the center of the mixing zone toward the upper and lower fronts, as shown by Fig. 18, where the profiles across the flow are plotted. A similar result was obtained from the spectral analysis performed by Cook et al. ${ }^{14}$ The energy is finally transferred toward the subfilter scales through the subgrid-scale dissipation $\varepsilon^{\mathrm{sg}}$. The dissipation caused by the numerical dealiasing filter described in Sec. III is $5.5 \%$ of $\varepsilon^{\mathrm{sg}}$ at $t=10 \tau$ and $5.0 \%$ at $t=15 \tau$. The input of energy by the buoyancy term goes directly into the Reynolds stress in the inhomogeneous direction, $R_{z z}$, and it is transferred by the pressure-strain correlation, $\Pi_{i j}^{r}=\left\langle\bar{p}^{\prime}\left(\bar{u}_{i, j}^{\prime}\right.\right.$ $\left.\left.+\bar{u}_{j, i}^{\prime}\right)\right\rangle$, toward the turbulent fluctuations in the homogeneous plane.

It is worth noticing as well that the subgrid-scale model shows backscatter (owing to the scale similar part) during the initial time, indicated by the positive values of $-\varepsilon^{\mathrm{sg}}$ for $t$ $<3 \tau$.

The curves are nondimensionalized with the quantity $\sqrt{(A g)^{3} \delta_{\rho}}$, formed by the length scale $\delta_{\rho}$ and the velocity scale $\sqrt{A g \delta_{\rho}}$. It is observed in Figs. 17 and 18 that the temporal evolution of the the buoyancy flux and the transport terms do not reach a plateau after the transient, though the subgrid-scale dissipation clearly does. This is not surprising since different statistical quantities achieve self-similarity at different times. Longer simulations would be interesting to ascertain this behavior.

Lastly, the integral of Eq. (104) along the vertical direction implies that the input of energy through the buoyancy flux is split between the gain of turbulent kinetic energy and the dissipation. Figure 19 shows the ratio

$$
\frac{\text { dissipation }}{\text { buoyancy production }}=\frac{\int\langle\bar{\rho}\rangle \varepsilon^{\mathrm{sg}} d z}{\int B^{r} d z} \text {. }
$$

Instead of the integral of $B^{r}$, the loss of potential energy based on the mean density profile is used in the literature. 


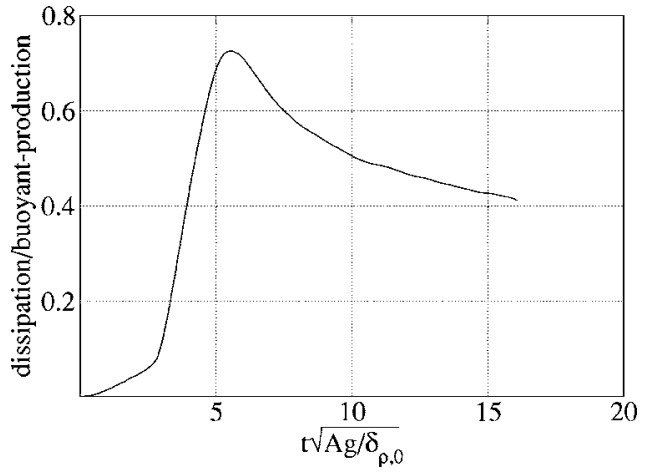

FIG. 19. Temporal evolution of the ratio of depth-integrated values of subgrid-scale dissipation and buoyancy production.

Both are equivalent when the domain of integration is closed, as obtained from mass conservation, but the present case allows outflow at the top and the bottom and the amount of potential energy released that goes into the turbulent motion is given by $B$, as indicated by Eq. (104). Figure 19 shows a tendency toward an asymptotic value which, however, is not achieved at the end of the simulation. The observed value, 0.42 , is slightly smaller than the value of 0.5 found in experiments ${ }^{11,16}$ and a recent numerical simulation. ${ }^{15}$

\section{CONCLUSIONS}

Compressibility effects in Rayleigh-Taylor turbulence with miscible fluids in an unbounded domain have been studied using analysis and LES. Three configurations are considered in the theoretical analysis. The first one is a two-layer system formed by a step-like distribution of the ratio between molecular weight and temperature. The density decreases exponentially with increasing height in each layer and each layer is buoyancy-stable. The second configuration has a density jump between two layers each being buoyancyneutral. The third configuration is defined by a step-like profile of the density itself, while the pressure decreases linearly with height in each layer. Each layer is buoyancy-unstable. It has been shown analytically that the turbulent Mach number is bounded from above, in the first two cases independently of the density jump at the interface and, for the last case, for moderate Atwood numbers $(A \leqslant 0.5)$. The reason is that the initial thermodynamic state of the system determines the amount of potential energy per unit mass involved in the turbulent mixing stage, and thus the level of turbulent fluctuations that is achievable is linked to the characteristic speed of sound such that the turbulent Mach number is limited.

In the particular case considered here of an ideal gas, this bound on the turbulent Mach number is derived to be $M_{t, \max } \simeq 0.25$ in the constant density configuration. However, this initial configuration in a compressible case is buoyancyunstable and may be difficult to set up. The bound is larger in the buoyancy-stable and buoyancy-neutral systems, for which $M_{t, \max } \simeq 0.6$, but the result is independent of the density ratio. It has to be noted that these values are conservative because the amount of potential energy that is dissipated (which is up to $50 \%$ in the incompressible case) is retained in the estimate of the turbulent kinetic energy. In all situations, $M_{t}$ is small enough so that compressibility effects may be relatively small. LES performed with a particular density jump at the interface of 3:1 for the stable and unstable configurations considered in the analysis indeed confirm that $M_{t}$ does not exceed the analytical bounds.

The compressibility effects have been studied in the LES decomposing the density fluctuation into the entropic part, due to variation of composition, and the acoustic part, due to intrinsic compressibility. This latter is found to be less than $10 \%$ of the total density fluctuation, indicating that the intrinsic compressibility effects are indeed small. Consequently, key features such as the quadratic time evolution of the mixing depth, the anisotropy of the Reynolds stresses, and the value of the mixing parameters compare well with those observed in the incompressible cases reported in the literature.

The LES is performed using a dynamic mixed model. The general evolution of the flow has been studied in the configuration with constant density. Mean profiles and mixing parameters are in good agreement with the available incompressible data to the extent that they can be compared. The structural changes in the flow as it evolves from the initial ordered finger-like structure to the disordered turbulent stage are manifest in practically all the discussed quantities. Peaks in density and velocity fluctuations, along with a minimum in mixing that corresponds to a more segregated state, are observed at times $t \simeq 2.5 \sqrt{\delta_{\rho, 0} /(A g)}$, where $\delta_{\rho, 0}$ is the initial thickness of the mixing region. Subsequently, the vorticity field, in the form of rings around the density fingers, starts to break the ordered density structures, mixing increases, and the subgrid model activates to provide the required dissipation.

The Reynolds stresses and the budget of the turbulent kinetic energy have been fully described. The level of anisotropy in the Reynolds stresses, measured as the ratio between the vertical to the horizontal fluctuations, is $\approx 1.8$. This is higher than that for shear driven flows, where the ratio of streamwise to cross-sectional fluctuations is closer to or smaller than unity. The vertical fluctuations gain energy from the available potential energy, and then there is a transfer to the horizontal fluctuations by the pressure-strain terms. This phenomenon peaks at the center (approximately) of the mixing width. The energy is then transported spatially toward the upper and the lower fronts of the turbulent core. Finally, the subgrid-scale dissipation transfers the energy toward the subfilter scales.

Although some statistics, notably the thickness of the mixing region, show signs of self-similarity, others continue to slowly evolve in time; the size of the problem, the initial thickness being $\approx 4 \%$ of the final value, is still too small for complete self-similarity.

\section{ACKNOWLEDGMENTS}

Partial support for J.P.M. was provided by Lawrence Livermore National Laboratory through the Student Employee Graduate Research Fellowship Program. This work 
was supported in part by a grant of HPC time from the Naval Oceanographic Office Department of Defense Major Shared Resource Center and by the National Partnership for Advanced Computational Infrastructure. We thank an anonymous reviewer for constructive comments.

${ }^{1}$ Lord Rayleigh, "Investigation of the character of the equilibrium of an incompressible heavy fluid of variable density," Proc. London Math. Soc. 14, 170 (1883).

${ }^{2}$ G. I. Taylor, "The instability of liquid surfaces when accelerated in in a direction perpendicular to their planes," Proc. R. Soc. London, Ser. A 201, 192 (1950).

${ }^{3}$ R. E. Duff, F. H. Harlow, and C. W. Hirt, "Effects of diffusion on interface instability between gases," Phys. Fluids 5, 417 (1962).

${ }^{4}$ D. Livescu, "Compressibility effects on the Rayleigh-Taylor instability growth between inmiscible fluid," Phys. Fluids 16, 118 (2004).

${ }^{5}$ K. I. Read, "Experimental investigation of turbulent mixing by RayleighTaylor instability," Physica D 12, 45 (1984).

${ }^{6}$ D. L. Youngs, "Numerical simulation of turbulent mixing by RayleighTaylor instability," Physica D 12, 32 (1984).

${ }^{7}$ D. H. Sharp, "An overview of Rayleigh-Taylor instability," Physica D 12, 3 (1984).

${ }^{8}$ D. L. Youngs, "Modelling turbulent mixing by Rayleigh-Taylor instability," Physica D 37, 270 (1989).

${ }^{9}$ Y. Zhou, "A scaling analysis of turbulent flows driven by Rayleigh-Taylor and Richtmyer-Meshkov instabilities," Phys. Fluids 13, 538 (2001).

${ }^{10}$ P. F. Linden and J. M. Redondo, "Molecular mixing in Rayleigh-Taylor instability. Part I: Global mixing," Phys. Fluids A 31269 (1991).

${ }^{11} \mathrm{P}$. Ramaprabhu and M. J. Andrews, "Experimental investigation of Rayleigh-Taylor mixing at small Atwood numbers," J. Fluid Mech. 502, 233 (2004)

${ }^{12}$ D. L. Youngs, "Three-dimensional numerical simulation of turbulent mixing by Rayleigh-Taylor instability," Phys. Fluids A 3, 1312 (1991).

${ }^{13}$ A. W. Cook and P. E. Dimotakis, "Transition stages of Rayleigh-Taylor instability between miscible fluids," J. Fluid Mech. 443, 69 (2001).

${ }^{14}$ A. W. Cook and Y. Zhou, "Energy transfer in Rayleigh-Taylor instability," Phys. Rev. E 66, 026312 (2002).

${ }^{15}$ A. W. Cook, W. Cabot, and P. L. Miller, "The mixing transition in rayleigh-taylor instability," J. Fluid Mech. 511, 333 (2004).

${ }^{16}$ P. F. Linden, J. M. Redondo, and D. L. Youngs, "Molecular mixing in Rayleigh-Taylor instability,” J. Fluid Mech. 265, 97 (1994).

${ }^{17}$ S. B. Dalziel, P. F. Linden, and D. L. Youngs, "Self-similarity and internal structure of turbulence induced by Rayleigh-Taylor instability," J. Fluid Mech. 399, 1 (1999).

${ }^{18}$ C. Meneveau and J. Katz, "Scale-invariance and turbulence models for large-eddy simulations," Annu. Rev. Fluid Mech. 32, 1 (2000).

${ }^{19}$ R. S. Rogallo and P. Moin, "Numerical simulation of turbulent flows," Annu. Rev. Fluid Mech. 16, 99 (1984).

${ }^{20} \mathrm{U}$. Piomelli and J. R. Chasnov, in Large-Eddy Simulations: Theory and Applications, Turbulence and Transition Modelling, edited by M. Hallbäck, D. S. Henningson, A. V. Johansson and P. H. Alfredsson (Kluwer, Dordrecht, 1996), pp. 269-336.

${ }^{21}$ M. Lesieur and O. Métais, "New trends if large-eddy simulation of turbulence," Annu. Rev. Fluid Mech. 28, 45 (1996).

${ }^{22}$ G. Dimonte, D. L. Youngs, A. Dimits, S. Weber, M. Marinak, S. Wunsch, C. Garasi, A. Robinson, M. J. Andrews, P. Ramaprabhu, A. C. Calder, B. Fryxell, J. Biello, L. Dursi, P. MacNeice, K. Olson, P. Ricker, R. Rosner, F. Timmes, H. Tufo, Y. Young, and M. Zingale, "A comparative study of the turbulent Rayleigh-Taylor instability using high-resolution threedimensional numerical simulations: The Alpha-group collaboration," Phys. Fluids 16, 1668 (2004).

${ }^{23}$ P. Chassaing, R. A. Antonia, F. Anselmet, L. Joly, and S. Sarkar, Variable Density Fluid Turbulence (Kluwer Academic, Dordrecht, 2002).

${ }^{24}$ S. Sarkar, G. Erlebacher, M. Y. Hussaini, and H. O. Kreiss, "The analysis and modeling of dilatational terms in compressible turbulence," J. Fluid Mech. 227, 473 (1991).
${ }^{25}$ S. Sarkar, "The stabilizing effect of compressibility in turbulent shear flow," J. Fluid Mech. 282, 163 (1995).

${ }^{26}$ A. W. Vreman, N. D. Sandham, and K. H. Luo, "Compressible mixing layer growth rate and turbulence characteristics," J. Fluid Mech. 320, 235 (1996).

${ }^{27}$ S. Sarkar, "On density and pressure fluctuations in uniformly sheared compressible flow," Proceedings, IUTAM Symposium on Variable Density Low-Speed Flows, Marseille, edited by L. Fulachier, J. L. Lumley, and F. Anselmet (Kluwer Academic, Dordrecht, 1996).

${ }^{28}$ J. B. Freund, S. K. Lele, and P. Moin, "Compressibility effects in a turbulent annular mixing layer. 1. Turbulence and growth rate," J. Fluid Mech. 421, 229 (2000).

${ }^{29}$ C. Pantano and S. Sarkar, "A study of compressibility effects in the highspeed, turbulent shear layer using direct simulation,” J. Fluid Mech., 451, 329 (2002).

${ }^{30}$ A. E. Gill, Atmosphere-Ocean Dynamics (Academic, New York, 1982).

${ }^{31}$ J. Pedloski, Geophysical Fluid Dynamics (Springer, Berlin, 1987).

${ }^{32}$ G. K. Batchelor, An Introduction to Fluid Dynamics (Cambridge University Press, Cambridge, 1967).

${ }^{33}$ Y. Chen, Y. Deng, J. Glimm, G. Li, and Q. Zang, "A renormalization group scaling analysis for compressible two-phase flow," Phys. Fluids A 5, 2929 (1993).

${ }^{34}$ B. Vreman, B. Geurts, and H. Kuerten, "Subgrid-modelling in LES of compressible flow," Appl. Sci. Res. 54, 191 (1995).

${ }^{35}$ B. Vreman, B. Geurts, and H. Kuerten, "Large-eddy simulation of the turbulent mixing layer," J. Fluid Mech. 339, 357 (1997).

${ }^{36}$ A. Yoshizawa, "Statistical theory for compressible turbulent shear flows, with application to subgrid modeling," Phys. Fluids 29, 2152 (1986).

${ }^{37}$ C. G. Speziale, G. Erlebacher, T. A. Zang, and M. Y. Hussaini, "The subgrid-scale modeling of compressible turbulence," Phys. Fluids 31, 940 (1988).

${ }^{38}$ P. Moin, K. Squires, W. Cabot, and S. Lee, "A dynamic subgrid-scale model for compressible turbulence and scalar transport," Phys. Fluids A 3, 2746 (1991).

${ }^{39}$ G. Erlebacher, M. Y. Hussaini, C. G. Speziale, and T. A. Zang, "Toward the large-eddy simulation of compressible flows," J. Fluid Mech. 238, 155 (1992).

${ }^{40}$ T. A. Zang, R. B. Dahlburg, and J. P. Dahlburg, "Direct and large-eddy simulation of three-dimensional compressible Navier-Stokes turbulence," Phys. Fluids A 4, 127 (1992).

${ }^{41}$ S. K. Lele, "Compact finite difference schemes with spectral-like resolution," J. Comput. Phys. 103, 16 (1992).

${ }^{42}$ F. K. Chow and P. Moin, "A further study of numerical errors in largeeddy simulations," J. Comput. Phys. 18, 366 (2003).

${ }^{43}$ J. H. Williamson, "Low-storage Runge-Kutta schemes," J. Comput. Phys. 35, 48 (1980).

${ }^{44} \mathrm{~K}$. W. Thompson, "Time-dependent boundary conditions for hyperbolic systems, II,” J. Comput. Phys. 89, 439 (1990).

${ }^{45}$ A. G. Kravchenko and P. Moin, "On the effect of numerical errors in large-eddy simulations of turbulent flows," J. Comput. Phys. 131, 310 (1997).

${ }^{46}$ Y. Morinishi, T. S. Lund, O. V. Vasilyev, and P. Moin, "Fully conservative higher order finite difference schemes for incompressible flows," J. Comput. Phys. 143, 90 (1998).

${ }^{47}$ G. A. Blaisdell, E. T. Spyropoulos, and J. H. Qin, "The effect of the formulation of nonlinear terms on aliasing errors in spectral methods," Appl. Numer. Math. 21, 207 (1996).

${ }^{48}$ C. Le Ribault, S. Sarkar, and S. A. Stanley, "Large eddy simulation of a plane jet," Phys. Fluids 11, 3069 (1999).

${ }^{49}$ C. Le Ribault, S. Sarkar, and S. A. Stanley, "Large eddy simulation of evolution of a passive scalar in plane jet," AIAA J. 391509 (2001).

${ }^{50}$ S. B. Pope, Turbulent Flows (Cambridge University Press, Cambridge, 2000).

${ }^{51}$ J. S. Bagget, J. Jiménez, and A. G. Kravchenko, "Resolution requirements in large-eddy simulations of shear flows," CTR Annual Research Briefs (Stanford University, Stanford, 1997). 